TRANSACTIONS OF THE

AMERICAN MATHEMATICAL SOCIETY

Volume 355, Number 2, Pages 837-864

S 0002-9947(02)03156-

Article electronically published on October 9, 2002

\title{
ANALYTIC MODELS FOR COMMUTING OPERATOR TUPLES ON BOUNDED SYMMETRIC DOMAINS
}

\author{
JONATHAN ARAZY AND MIROSLAV ENGLIŠ
}

\begin{abstract}
For a domain $\Omega$ in $\mathbb{C}^{d}$ and a Hilbert space $\mathcal{H}$ of analytic functions on $\Omega$ which satisfies certain conditions, we characterize the commuting $d$-tuples $T=\left(T_{1}, \ldots, T_{d}\right)$ of operators on a separable Hilbert space $H$ such that $T^{*}$ is unitarily equivalent to the restriction of $M^{*}$ to an invariant subspace, where $M$ is the operator $d$-tuple $Z \otimes I$ on the Hilbert space tensor product $\mathcal{H} \otimes H$. For $\Omega$ the unit disc and $\mathcal{H}$ the Hardy space $H^{2}$, this reduces to a well-known theorem of Sz.-Nagy and Foias; for $\mathcal{H}$ a reproducing kernel Hilbert space on $\Omega \subset \mathbb{C}^{d}$ such that the reciprocal $1 / K(x, \bar{y})$ of its reproducing kernel is a polynomial in $x$ and $\bar{y}$, this is a recent result of Ambrozie, Müller and the second author. In this paper, we extend the latter result by treating spaces $\mathcal{H}$ for which $1 / K$ ceases to be a polynomial, or even has a pole: namely, the standard weighted Bergman spaces (or, rather, their analytic continuation) $\mathcal{H}=\mathcal{H}_{\nu}$ on a Cartan domain corresponding to the parameter $\nu$ in the continuous Wallach set, and reproducing kernel Hilbert spaces $\mathcal{H}$ for which $1 / K$ is a rational function. Further, we treat also the more general problem when the operator $M$ is replaced by $M \oplus W, W$ being a certain generalization of a unitary operator tuple. For the case of the spaces $\mathcal{H}_{\nu}$ on Cartan domains, our results are based on an analysis of the homogeneous multiplication operators on $\Omega$, which seems to be of an independent interest.
\end{abstract}

Let $T$ be a bounded linear operator on a separable Hilbert space $H$. A well-known result of Sz.-Nagy and Foias says that the following two assertions are equivalent:

(a) $I-T T^{*} \geq 0$ (i.e., $T$ is a contraction) and $T^{* n} \rightarrow 0$

in the strong operator topology;

(b) $T^{*}$ is unitarily equivalent to the restriction of a backward shift of infinite multiplicity to an invariant subspace.

There is also a somewhat stronger form of this result which deals with the case when the second half of the condition (a) is not satisfied: namely,

$T$ (equivalently, $T^{*}$ ) is a contraction if and only if $T^{*}$ is unitarily equivalent (0.2) to the restriction to an invariant subspace of the direct sum of a backward shift of infinite multiplicity and a unitary operator.

Received by the editors February 14, 2002.

2000 Mathematics Subject Classification. Primary 47A45; Secondary 47A13, 32M15, 32A07.

Key words and phrases. Coanalytic models, reproducing kernels, bounded symmetric domains, commuting operator tuples, functional calculus.

The second author's research was supported by GA ČR grant no. 201/00/0208 and GA AV ČR grant no. A1019005. The second author was also partially supported by the Israeli Academy of Sciences during his visit to Haifa in January 2001, during which part of this work was done. 
A number of generalizations of the Sz.-Nagy-Foias theorem have subsequently been established by several authors. Typically, in these results the operator $T$ is replaced by a $d$-tuple of commuting operators $T_{1}, \ldots, T_{d}$ on $H$, the contractivity of $T$ by an appropriate positivity condition on the tuple $T_{1}, \ldots, T_{d}$ (e.g. the spherical, or row, contractivity $I-T_{1} T_{1}^{*}-\cdots-T_{d} T_{d}^{*} \geq 0$ ), and the backward shift by the $d$-tuple of the adjoints of the operators of multiplication by the coordinates in a suitable Hilbert space $\mathcal{H}$ of analytic functions. The first result of this kind is due to Agler Ag1, Ag2, for $d=1$ and $\mathcal{H}$ the weighted Bergman space on the unit disc $\mathbb{D}$ with reproducing kernel $(1-z \bar{w})^{-m}, m=1,2,3, \ldots$ The spherical contractions were considered by Drury [Dr], Athavale [At1, At3] and Müller and Vasilescu [MV], whose results correspond to $\mathcal{H}$ the weighted Bergman space on the unit ball $\mathbb{B}^{d}$ of $\mathbb{C}^{d}$ with reproducing kernel $(1-\langle z, w\rangle)^{-m}, m=1,2,3, \ldots$ (See also Arv for a farreaching operator-algebraic development of this theory.) In Pott [Po] these results were further extended to the case of $\mathcal{H}$ the Hilbert space with the reproducing kernel $\left(1-\langle z, w\rangle-P\left(z_{1} \bar{w}_{1}, \ldots, z_{d} \bar{w}_{d}\right)\right)^{-m}, m=1,2, \ldots$, on a Reinhardt domain of the form $\left\{z \in \mathbb{C}^{d}: P\left(\left|z_{1}\right|^{2}, \ldots,\left|z_{d}\right|^{2}\right)<1-\|z\|^{2}\right\}$, where $P$ is a polynomial with positive coefficients and without constant and linear terms. Finally, the most general result of this type has recently been obtained by Ambrozie, Müller and the second author in AEM]. Namely, let $\Omega$ be a domain in $\mathbb{C}^{d}$ and $\mathcal{H}$ a Hilbert space of analytic functions on $\Omega$ which has the following properties:

(1) $\mathcal{H}$ is invariant under the operators $Z_{j}$ of multiplications by the coordinate functions $z_{j}(j=1, \ldots, d)$.

(2) The evaluation functionals are continuous on $\mathcal{H}$. Consequently, there exists a reproducing kernel $K(z, \bar{w})$ for $\mathcal{H}$.

(3) $\mathcal{H}$ contains all polynomials and they are dense in it.

(4) The function $1 / K(z, \bar{w})$ is a polynomial (in $z$ and $\bar{w}$ ).

It is well known that for any orthonormal basis $\left\{\psi_{k}\right\}$ of $\mathcal{H}$, the reproducing kernel is given by

$$
K(z, \bar{w})=\sum_{k} \psi_{k}(z) \overline{\psi_{k}(w)} .
$$

In view of (3), by applying the Gramm-Schmidt orthogonalization process, we may construct an orthonormal basis $\left\{\psi_{k}\right\}$ consisting of polynomials and such that, conversely, any polynomial is a linear combination of a finite number of the $\psi_{k}$. We fix such a basis from now on. For each $m$, set

$$
f_{m}(z, \bar{w})=\sum_{k \geq m} \frac{\psi_{k}(z) \overline{\psi_{k}(w)}}{K(z, \bar{w})} .
$$

Then $f_{0}(z, \bar{w}) \equiv 1$ on $\Omega \times \Omega$. By virtue of (4) and our choice of the basis, the difference $f_{0}-f_{m}$ is a polynomial in $z, \bar{w}$, for each $m$; thus the $f_{m}$ themselves are, in fact, polynomials.

For a commuting $d$-tuple $T=\left(T_{1}, \ldots, T_{d}\right)$ of operators and a polynomial $p(z, \bar{w})$ of $z, \bar{w} \in \mathbb{C}^{d}$, holomorphic in $z$ and $\bar{w}$, let us define (the "Maslov calculus" [M], [MN])

$$
p\left(T, T^{*}\right):=\sum_{\alpha, \beta} p_{\alpha \beta} T^{\alpha} T^{* \beta} \quad \text { if } \quad p(z, \bar{w})=\sum_{\alpha, \beta} z^{\alpha} \bar{w}^{\beta} .
$$

(Up to the order of $T$ and $T^{*}$, this coincides with the "hereditary calculus" of Agler [Ag1.) The main result of [AEM] is the following. 
Theorem ([AEM], Corollary 15). Assume that $\mathcal{H}$ satisfies $(1)-(4)$ and let $T$ be a commuting d-tuple of operators on a separable Hilbert space $H$. Denote by $M$ the operator d-tuple $Z \otimes I$ on the Hilbert space tensor product $\mathcal{H} \otimes H$. Then the following are equivalent:

(a) $T^{*}$ is unitarily equivalent to the restriction of $M^{*}$ to an invariant subspace.

(b) $\frac{1}{K}\left(T, T^{*}\right) \geq 0$ and $\left\langle f_{m}\left(T, T^{*}\right) h, h\right\rangle \rightarrow 0 \forall h \in H$.

Note that the operator $Z \otimes I$ is nothing but the direct sum of $m$ copies of $Z$, where $m=\operatorname{dim} H \leq \aleph_{0}$. We will use these two interpretations interchangeably in the sequel without further mention.

Note also that the property in part (b) depends on the choice of the orthonormal basis $\left\{\psi_{k}\right\}$, but the property in (a) does not.

For $\Omega=\mathbb{D}$, the unit disc, $\mathcal{H}=H^{2}$, the Hardy space with the reproducing kernel $K(z, \bar{w})=1 /(1-z \bar{w})$, so that $\frac{1}{K}\left(T, T^{*}\right)=I-T T^{*}$, and $\psi_{k}(z)=z^{k}$ the standard orthonormal basis, we get $f_{m}\left(T, T^{*}\right)=T^{m} T^{* m}$, the operator $M$ is just the forward shift of infinite multiplicity, and one thus recovers the result of Sz.-Nagy and Foias (0.1) mentioned in the beginning. Similarly one can obtain by suitable choices of $\mathcal{H}$ the other generalizations of $(0.1)$ mentioned above.

In spite of the fairly general character of the last theorem, the assumption (4) is still quite restrictive. Even in the most familiar case, the weighted Bergman spaces on the unit disc with respect to the weights $\left(1-|z|^{2}\right)^{\alpha}, \alpha>-1$ (so that the reproducing kernels are $\left.\frac{\alpha-1}{\pi}(1-z \bar{w})^{-\alpha-2}\right)$, it is satisfied only for $\alpha$ an integer. Similarly, for $\Omega$ a smoothly bounded strictly pseudoconvex domain in $\mathbb{C}^{2}$ and $\mathcal{H}$ the Bergman space on $\Omega$ with respect to the Lebesgue measure, it follows from the results of Fefferman [Fe] and Graham [Gh, Theorem 3.2] that (4) can only be satisfied if $\Omega$ is biholomorphically equivalent to the unit ball. In this paper, we therefore present several extensions of the last theorem to cases when (4) is not fulfilled. It turns out that the main problem is, as can be expected, to make sense of the expression $\frac{1}{K}\left(T, T^{*}\right)$ when $\frac{1}{K}$ is not a polynomial, or, more generally, a holomorphic function of $z, \bar{w}$ in a neighbourhood of the Taylor spectrum of the $d$-tuple $T$. We remark that, curiously enough, it is frequently possible to make sense of $\frac{1}{K}\left(T, T^{*}\right)$ even if there exist $x, y \in \Omega$, belonging to the Taylor spectrum, for which $K(x, \bar{y})=0$; cf. Section 4 below.

The paper is organized as follows. In Section 1, we show that, loosely speaking, the above theorem remains in force even without the assumption (4), as long as one can "make sense of the operator $\frac{1}{K}\left(T, T^{*}\right)$ in a reasonable way" (Theorem 1.3). (In fact, it turns out that we can even dispense with the hypothesis (2) as well.) For want of a better term, we will call any "reasonable way of making sense of $\frac{1}{K}\left(T, T^{*}\right)$ " a $\frac{1}{K}$-calculus for the functional Hilbert space $\mathcal{H}$; see below for the precise definition. In Section 2 we review some background material on bounded symmetric domains. The next two sections give some examples of $\frac{1}{K}$-calculi. In Section 3 we construct a $\frac{1}{K}$-calculus for $\mathcal{H}$ the standard weighted Bergman space on a Cartan (i.e., irreducible bounded symmetric) domain in $\mathbb{C}^{d}$ corresponding to the parameter $\nu$ in the Wallach set (Proposition 3.1 and Theorem 3.3). The results are based on an analysis of the multiplication operators on these spaces which we believe to be of an independent interest. In Section 4 we deal with the case when the reproducing kernel of $\mathcal{H}$ is a rational function. The last two sections are concerned with analogues of (0.2), i.e., descriptions of the " $\frac{1}{K}$-unitary part": in Section 5, an analogue of $(0.2)$ is proved for rotation-invariant functional Hilbert spaces $\mathcal{H}$ on 
complete Reinhardt domains $\Omega \subset \mathbb{C}^{d}$ (Theorem 5.1), and includes as a special case the above-mentioned results of Pott [Po]. Finally, in Section 6 we also establish an analogue of $(0.2)$ for the weighted Bergman spaces on Cartan domains from Section 3 (Theorems 6.3 and 6.4).

Throughout the paper, $\mathcal{H}$ stands for a Hilbert space of holomorphic functions on a domain $\Omega \subset \mathbb{C}^{d}$ which satisfies the condition (1) above (but not necessarily $(2),(3)$ or $(4))$. For a separable Hilbert space $H$, we denote by $\mathcal{B}(\mathcal{H})$ the space of all bounded linear operators on $H$, by $\mathcal{B}(\mathcal{B}(\mathcal{H}))$ the space of all bounded linear operators (transformers) on the Banach space $\mathcal{B}(\mathcal{H})$, and by $\mathcal{B}(\mathcal{H})^{+}$the cone of nonnegative operators in $\mathcal{B}(\mathcal{H})$ (i.e., $T \in \mathcal{B}(\mathcal{H})^{+}$if $\langle T h, h\rangle \geq 0 \forall h \in H$ ). The symbol $I_{H}$ stands for the identity operator on $H$; the subscript $H$ will be sometimes omitted. For $T, S \in \mathcal{B}(\mathcal{H})$, we denote by $L_{T}: X \mapsto T X$ and $R_{S}: X \mapsto X S$ the corresponding operators of left and right multiplication by $T$ and $S$, respectively. Similarly, for commuting $d$-tuples $T=\left(T_{1}, \ldots, T_{d}\right)$ and $S=\left(S_{1}, \ldots, S_{d}\right)$ of operators in $\mathcal{B}(\mathcal{H})$ we denote by $L_{T}$ and $R_{S}$ the $d$-tuples $\left(L_{T_{1}}, \ldots, L_{T_{d}}\right)$ and $\left(R_{S_{1}}, \ldots, R_{S_{d}}\right)$, respectively. Note that $\left(L_{T}, R_{S}\right)$ is a commuting $2 d$-tuple of elements of $\mathcal{B}(\mathcal{B}(\mathcal{H}))$. The polynomial ("Maslov") calculus above can thus be rewritten as

$$
p\left(T, T^{*}\right)=p\left(L_{T}, R_{T^{*}}\right) I,
$$

where the right-hand side is simply the polynomial of the commuting operators $L_{T}$ and $R_{T^{*}}$, applied to the identity operator $I$, i.e., $p\left(L_{T}, R_{T^{*}}\right) \in \mathcal{B}(\mathcal{B}(\mathcal{H}))$ is obtained from $p(z, \bar{w})$ upon substituting the commuting operators $L_{T_{j}}$ and $R_{T_{j}^{*}}$ for $z_{j}$ and $\bar{w}_{j}$, respectively. Finally, $Z$ will always stand for the operator tuple of multiplications by the coordinate functions on $\mathcal{H}, P_{0}$ for the operator

$$
\left.P_{0}:=\langle\cdot, \mathbf{1}\rangle \mathbf{1} \quad \text { (i.e. } P_{0} x=\langle x, \mathbf{1}\rangle \mathbf{1}\right)
$$

on $\mathcal{H}$, and the operator $Z \otimes I$ will be occasionally denoted by $M$.

\section{1. $\frac{1}{K}$-CALCULI}

Definition 1.1. Let $\mathcal{H}$ be a Hilbert space of holomorphic functions on a domain $\Omega \subset \mathbb{C}^{d}$ which satisfies the condition (1) and contains the constant functions. We say that $\mathcal{H}$ admits a $\frac{1}{K}$-calculus if for each separable Hilbert space $H$ there exists a (nonlinear) mapping $\Phi_{H}$, defined on a subset $\operatorname{dom} \Phi_{H} \subset \mathcal{B}(\mathcal{H})$ and having as values subsets of $\mathcal{B}(\mathcal{H})^{+}$, such that the following two conditions are satisfied:

$(\alpha)$ if $V: H_{1} \rightarrow H_{2}$ is an isometry and $T_{1} V^{*}=V^{*} T_{2}$, then $T_{2} \in \operatorname{dom} \Phi_{H_{2}}$ implies that $T_{1} \in \operatorname{dom} \Phi_{H_{1}}$ and $\Phi_{H_{1}}\left(T_{1}\right) \supset V^{*} \Phi_{H_{2}}\left(T_{2}\right) V$;

$(\beta)$ for any separable Hilbert space $E$, the operator $Z \otimes I$ on $\mathcal{H} \otimes E$ belongs to $\operatorname{dom} \Phi_{\mathcal{H} \otimes E}$ and $\Phi_{\mathcal{H} \otimes E}(Z \otimes I)$ is the subset of $\mathcal{B}(H \otimes E)$ consisting of the single element $P_{0} \otimes I_{E}$.

We say that the $\frac{1}{K}$-calculus $\Phi$ is strong if $\Phi_{H}(T)$ consists of a single element, for any $H$ and $T \in \operatorname{dom} \Phi_{H}$.

For strong $\frac{1}{K}$-calculi, we will usually not distinguish between the set $\Phi_{H}(T)$ and the single element it contains; in particular, we will abuse the notation and denote both by $\Phi_{H}(T)$.

The idea behind the $\frac{1}{K}$-calculus is that one should think of $\Phi_{H}(T)$ as "all possible values of $\frac{1}{K}\left(T, T^{*}\right)^{\text {" where }} K(z, \bar{w})$ is the reproducing kernel of $\mathcal{H}$. This is elucidated by the next proposition. 
Proposition 1.2. Assume that the Hilbert space $\mathcal{H}$ has reproducing kernel $K(z, \bar{w})$ such that $1 / K(z, \bar{w})$ is a polynomial in $z$ and $\bar{w}$. Then the recipe

$$
\Phi_{H}(T)=\frac{1}{K}\left(T, T^{*}\right) \quad\left(\equiv \frac{1}{K}\left(L_{T}, R_{T^{*}}\right) I_{H}\right),
$$

with $T \in \operatorname{dom} \Phi_{H}$ if and only if the right-hand side is a nonnegative operator, gives a strong $\frac{1}{K}$-calculus for $\mathcal{H}$.

Proof. Since $1 / K(z, \bar{w})$ is a polynomial in $z$ and $\bar{w}, \frac{1}{K}\left(L_{T}, R_{T^{*}}\right)$ makes sense for any commuting tuple $T$, and the validity of $(\alpha)$ in Definition 1.1 follows from the properties of the polynomial functional calculus. (In fact, it would be enough to assume that $1 / K(z, \bar{w})$ is an entire function of $z$ and $\bar{w}$.) It also follows from the latter that $\Phi_{\mathcal{H} \otimes H}(T \otimes I)=\Phi_{H}(T) \otimes I$; so to prove $(\beta)$ it suffices to show that $\Phi_{\mathcal{H}}(Z)=P_{0}$. This was proved in Proposition 13 in AEM] for convenience, we reproduce the proof here.

Recall that for any $w \in \Omega$,

$$
Z_{j}^{*} K_{w}=\bar{w}_{j} K_{w}
$$

where $K_{w}(z):=K(z, \bar{w})$. It follows that for any polynomial $p(z, \bar{w})$ and $x, y \in \Omega$,

$$
\left\langle p\left(Z, Z^{*}\right) K_{y}, K_{x}\right\rangle=p(x, \bar{y})\left\langle K_{y}, K_{x}\right\rangle=p(x, \bar{y}) K(x, \bar{y}) .
$$

So, in particular, $\left\langle\frac{1}{K}\left(Z, Z^{*}\right) K_{y}, K_{x}\right\rangle=1 \forall x, y \in \Omega$. Since also $\left\langle K_{y}, \mathbf{1}\right\rangle\left\langle\mathbf{1}, K_{x}\right\rangle=$ $\overline{\mathbf{1}(y)} \mathbf{1}(x)=1 \forall x, y \in \Omega$, and since the span of $\left\{K_{w}\right\}_{w \in \Omega}$ is dense in $\mathcal{H}$, it follows that $\frac{1}{K}\left(Z, Z^{*}\right)=P_{0}$.

Let us now assume that $\mathcal{H}$ satisfies the conditions (1) and (3). We fix an orthonormal basis $\boldsymbol{\psi}=\left\{\psi_{j}\right\}_{j=0}^{\infty}$ of $\mathcal{H}$ such that each $\psi_{j}$ is a polynomial and, conversely, every polynomial is a linear combination of finitely many $\psi_{j}$ 's; as noted in the introduction, the existence of such a basis is a consequence of the condition (3). For any nonnegative operator $C$ and a commuting $d$-tuple $T$ of operators in $\mathcal{B}(\mathcal{H})$, set

$$
f_{m, C, \boldsymbol{\psi}}(T)=I-\sum_{0 \leq j<m} \psi_{j}(T) C \psi_{j}(T)^{*} .
$$

We will sometimes suppress the subscripts $C$ and $\boldsymbol{\psi}$ as well as the argument $T$ when no confusion can arise.

The following theorem is the main result of this section.

Theorem 1.3. Let $\mathcal{H}$ be a Hilbert space of holomorphic functions on a domain $\Omega \subset \mathbb{C}^{d}$ that satisfies the conditions (1) and (3). Then for a commuting d-tuple $T$ of operators on a separable Hilbert space $H$, the following assertions are equivalent:

(a) $T^{*}$ is unitarily equivalent to the restriction of $Z^{*} \otimes I_{E}$, where $E$ is a separable Hilbert space, to an invariant subspace;

(b) there exists a nonnegative operator $C$ in $\mathcal{B}(\mathcal{H})$ such that $\left\langle f_{m, C}(T) h, h\right\rangle \rightarrow 0$ as $m \rightarrow \infty \forall h \in H$ (equivalently, $\left\|f_{m, C}(T) h\right\| \rightarrow 0 \forall h \in H$ ).

If $\mathcal{H}$ admits a $\frac{1}{K}$-calculus, then (a) and (b) are also equivalent to

(c) $T \in \operatorname{dom} \Phi_{H}$ and the assertion (b) holds for some $C \in \Phi_{H}(T)$.

Proof. (a) $\Longrightarrow$ (b). Denote, for brevity, $M:=Z \otimes I_{E}$. Let $V: H \rightarrow \mathcal{H} \otimes E$ be an isometry such that $V T^{*}=M^{*} V$. Set

$$
C:=V^{*}\left(P_{0} \otimes I_{E}\right) V
$$


Then a simple calculation shows that

$$
\begin{aligned}
f_{m, C}(T) & =I-\sum_{0 \leq j<m} \psi_{j}(T) V^{*}\left(P_{0} \otimes I_{E}\right) V \psi_{j}(T)^{*} \\
& =I-\sum_{0 \leq j<m} V^{*} \psi_{j}(M)\left(P_{0} \otimes I_{E}\right) \psi_{j}(M)^{*} V \\
& =I-\sum_{0 \leq j<m} V^{*}\left[\psi_{j}(Z) P_{0} \psi_{j}(Z)^{*} \otimes I_{E}\right] V \\
& =I-\sum_{0 \leq j<m} V^{*}\left(\left\langle\cdot, \psi_{j}\right\rangle \psi_{j} \otimes I_{E}\right) V \\
& =V^{*}\left[\left(I-\sum_{0 \leq j<m}\left\langle\cdot, \psi_{j}\right\rangle \psi_{j}\right) \otimes I_{E}\right] V .
\end{aligned}
$$

However, since $\left\{\psi_{j}\right\}$ is an orthonormal basis of $\mathcal{H}$, the last sum tends to the identity operator in the strong operator topology. Thus even $f_{m, C}(T) \rightarrow 0$ strongly.

(a) $\Longrightarrow$ (c). If $\mathcal{H}$ admits a $\frac{1}{K}$-calculus, then the operator (1.4) must belong to $\Phi_{H}(T)$ by Definition 1.1.

(c) $\Longrightarrow$ (b). Trivial.

(b) $\Longrightarrow$ (a). Define an operator $V: H \rightarrow \mathcal{H} \otimes H$ by

$$
V h=\sum_{k} \psi_{k}(z) \otimes C^{1 / 2} \psi_{k}(T)^{*} h .
$$

We claim that $V$ is well-defined (i.e., the sum converges) and is, in fact, an isometry satisfying $V T^{*}=M^{*} V$.

To see that $V$ is well-defined and an isometry, observe that for any $k<m$ and $h \in H$,

$$
\begin{aligned}
\left\|\sum_{k \leq j<m} \psi_{j}(z) \otimes C^{1 / 2} \psi_{j}(T)^{*} h\right\|^{2} & =\sum_{k \leq j<m}\left\|C^{1 / 2} \psi_{j}(T)^{*} h\right\|^{2} \\
& =\sum_{k \leq j<m}\left\langle\psi_{j}(T) C \psi_{j}(T)^{*} h, h\right\rangle \\
& =\left\langle\left(f_{k}-f_{m}\right) h, h\right\rangle .
\end{aligned}
$$

Since $\left\langle f_{m} h, h\right\rangle \rightarrow 0$ by hypothesis, it follows that the partial sums of the right-hand side of (1.5) form a Cauchy sequence, and letting $k=0$ and $m \rightarrow \infty$ shows that $\|V h\|^{2}=\left\langle f_{0} h, h\right\rangle=\|h\|^{2}$, i.e., $V$ is an isometry.

To prove that $V T^{*}=M^{*} V$, observe that $\forall h, h^{\prime} \in H$ and any $k$,

$$
\left\langle V h, \psi_{k} \otimes h^{\prime}\right\rangle=\left\langle C^{1 / 2} \psi_{k}(T)^{*} h, h^{\prime}\right\rangle=\left\langle h, \psi_{k}(T) C^{1 / 2} h^{\prime}\right\rangle .
$$

So, by virtue of our choice of the basis $\psi_{k}$,

$$
\left\langle V h, f \otimes h^{\prime}\right\rangle=\left\langle h, f(T) C^{1 / 2} h^{\prime}\right\rangle
$$

for any polynomial $f$. Thus

$$
\begin{aligned}
\left\langle V T_{j}^{*} h, \psi_{k} \otimes h^{\prime}\right\rangle & =\left\langle T_{j}^{*} h, \psi_{k}(T) C^{1 / 2} h^{\prime}\right\rangle=\left\langle h, T_{j} \psi_{k}(T) C^{1 / 2} h^{\prime}\right\rangle \\
= & \left\langle h,\left(z_{j} \psi_{k}\right)(T) C^{1 / 2} h^{\prime}\right\rangle=\left\langle V h, z_{j} \psi_{k} \otimes h^{\prime}\right\rangle=\left\langle M_{j}^{*} V h, \psi_{k} \otimes h^{\prime}\right\rangle,
\end{aligned}
$$

and the assertion follows. 
We now turn to the question of existence of $\frac{1}{K}$-calculi. The following proposition is immediate from the definition of $\frac{1}{K}$-calculus:

Proposition 1.4. Let $\mathcal{H}$ be a Hilbert space of holomorphic functions satisfying the condition (1) and containing the constant functions. Then for $\mathcal{H}$ to admit a $\frac{1}{K}$-calculus, it is necessary and sufficient that for any two separable Hilbert spaces $E_{1}, E_{2}$ and an isometry $V: \mathcal{H} \otimes E_{1} \rightarrow \mathcal{H} \otimes E_{2}$,

$$
\left(Z \otimes I_{E_{2}}\right)^{*} V=V\left(Z \otimes I_{E_{1}}\right)^{*} \quad \text { implies } \quad V^{*}\left(P_{0} \otimes I_{E_{2}}\right) V=P_{0} \otimes I_{E_{1}} .
$$

If this is the case, then a $\frac{1}{K}$-calculus $\Psi$ can be defined by

$$
\begin{gathered}
\operatorname{dom} \Psi_{H}=\{T: \text { there exists a separable Hilbert space } E \text { and an isometry } \\
\left.V: H \rightarrow \mathcal{H} \otimes E \text { such that } V T^{*}=\left(Z \otimes I_{E}\right)^{*} V\right\} ; \\
\Psi_{H}(T)=\left\{V^{*}\left(P_{0} \otimes I_{E}\right) V, \text { for all } V \text { as above }\right\} .
\end{gathered}
$$

This $\frac{1}{K}$-calculus is "minimal" in the sense that for any other $\frac{1}{K}$-calculus $\Phi$, one has $\operatorname{dom} \Phi_{H} \supset \operatorname{dom} \Psi_{H}$ for any $H$ and $\Phi_{H}(T) \supset \Psi_{H}(T) \forall T \in \operatorname{dom} \Psi_{H}$.

Further, the following two assertions are equivalent:

(a) there exists a strong $\frac{1}{K}$-calculus for $\mathcal{H}$;

(b) for any three separable Hilbert spaces $H, E_{1}, E_{2}$, operator $T \in \mathcal{B}(\mathcal{H})$ and isometries $V_{j}: H \rightarrow \mathcal{H} \otimes E_{j}$ satisfying $\left(Z \otimes I_{E_{j}}\right)^{*} V_{j}=V_{j} T^{*}(j=1,2)$, it follows that

$$
V_{1}^{*}\left[P_{0} \otimes I_{E_{1}}\right] V_{1}=V_{2}^{*}\left[P_{0} \otimes I_{E_{2}}\right] V_{2} .
$$

The question of existence of a $\frac{1}{K}$-calculus for any Hilbert space $\mathcal{H}$ of holomorphic functions satisfying (1) and containing the constant functions seems to be an interesting open problem. Observe that for $V_{1}=I,(1.8)$ reduces just to the consistency condition (1.7) (with $V=V_{2}$ ). We finish this section by exhibiting a large class of spaces $\mathcal{H}$ for which the latter is fulfilled.

Proposition 1.5. Let $\mathcal{H}$ be a Hilbert space of holomorphic functions satisfying the conditions (1) and (2) and containing the constant functions. Assume that for any $w \in \Omega$,

$$
\operatorname{dim} \operatorname{ker}(Z-w)^{*}=1
$$

(that is, $\operatorname{dim} \bigcap_{j=1}^{d} \operatorname{ker}\left(Z_{j}-w_{j}\right)^{*}=1$ ). Then (1.7) holds (and, consequently, $\mathcal{H}$ admits a $\frac{1}{K}$-calculus).

Proof. Note that from the hypothesis that $\mathcal{H}$ contain the constant functions, it follows that $K(w, \bar{w})>0 \forall w \in \Omega$ (because $1=\mathbf{1}(w) \leq\|\mathbf{1}\| K(w, \bar{w})^{1 / 2}$ by CauchySchwarz). Since the functions $K_{w}:=K(\cdot, \bar{w})$ satisfy (1.1), (1.9) implies that $\operatorname{ker}(Z-$ $w)^{*}=\mathbb{C} K_{w}, \forall w \in \Omega$. It follows that $\operatorname{ker}\left[(Z-w) \otimes I_{E}\right]^{*}=K_{w} \otimes E$ for all $w \in \Omega$, for any Hilbert space $E$. (Indeed, if $\left\{e_{j}\right\}$ is an orthonormal basis of $E$, then any element of $\mathcal{H} \otimes E$ can be written uniquely in the form $\sum_{j} f_{j} \otimes e_{j}$ with $f_{j} \in \mathcal{H}$, and $\left[(Z-w)^{*} \otimes I\right] \sum_{j} f_{j} \otimes e_{j}=\sum_{j}(Z-w)^{*} f_{j} \otimes e_{j}=0$ if and only if $(Z-w)^{*} f_{j}=0 \forall j$, or $f_{j}=c_{j} K_{w} \forall j$ with some $c_{j} \in \mathbb{C}$, whence $\sum_{j} f_{j} \otimes e_{j}=K_{w} \otimes \sum_{j} c_{j} e_{j} \in K_{w} \otimes E$.) Now for $f \in E_{1},\left(Z \otimes I_{E_{2}}\right)^{*} V=V\left(Z \otimes I_{E_{1}}\right)^{*}$ implies

$$
\left(Z \otimes I_{E_{2}}\right)^{*} V\left(K_{w} \otimes f\right)=V\left(\bar{w} K_{w} \otimes f\right)=\bar{w} V\left(K_{w} \otimes f\right),
$$

and so $V\left(K_{w} \otimes f\right) \in \operatorname{ker}\left[(Z-w) \otimes I_{E_{2}}\right]^{*}$; hence,

$$
V\left(K_{w} \otimes f\right)=K_{w} \otimes C_{w} f, \quad \forall w \in \Omega
$$


for some operators $C_{w} \in \mathcal{B}\left(E_{1}, E_{2}\right)$. Since $K_{w}$ depends continuously on $w$, it follows from the boundedness of $V$ that $K_{w} \otimes C_{w} f \rightarrow K_{z} \otimes C_{z} f$ as $w \rightarrow z$, and it transpires that the mapping $w \mapsto C_{w}$ is continuous in the strong operator topology. The condition that $V$ be an isometry, i.e., $V^{*} V=I$, translates into $C_{z}^{*} C_{w}=I$ for all $z, w \in \Omega$ for which $K(z, \bar{w}) \neq 0$; hence, for all $z, w \in \Omega$ by strong continuity. Thus $\left(C_{z}^{*}-C_{w}^{*}\right)\left(C_{z}-C_{w}\right)=C_{z}^{*} C_{z}-C_{z}^{*} C_{w}-C_{w}^{*} C_{z}+C_{w}^{*} C_{w}=I-I-I+I=0$. So $C_{z}-C_{w}=0$; hence, $C_{z}=C_{w}=: C$ is independent of $z$. Since the linear span of $K_{w}, w \in \Omega$, is dense in $\mathcal{H}$, we thus see that $V=I \otimes C$, with $C^{*} C=I$, and (1.7) immediately follows.

Theorem 1.6. Let $\mathcal{H}$ be a Hilbert space of holomorphic functions satisfying (1), (2) and (3). Then (1.9) holds for all $w \in \Omega$. Consequently, $\mathcal{H}$ admits a $\frac{1}{K}$-calculus.

Proof. Let $\mathcal{M}$ be the closed linear span in $\mathcal{H}$ of the subspaces $\operatorname{Ran}\left(Z_{j}-w_{j}\right), j=$ $1, \ldots, d$; we need to show that $\mathcal{M}$ is of codimension 1 . Let $f \perp \mathcal{M}$. By (3), there exist $a_{k} \in \mathbb{C}$ and $X_{k} \in \mathcal{M}(k=1,2, \ldots)$ such that $a_{k} \mathbf{1}+X_{k} \rightarrow f$. Applying $I-P_{\mathcal{M}}$, where $P_{\mathcal{M}}$ is the orthogonal projection in $\mathcal{H}$ onto $\mathcal{M}$, we get $a_{k}\left(I-P_{\mathcal{M}}\right) \mathbf{1} \rightarrow f$. Thus any $f \in \mathcal{M}^{\perp}$ is a multiple of $\left(I-P_{\mathcal{M}}\right) \mathbf{1}$; consequently, $\operatorname{dim} \mathcal{M}^{\perp} \leq 1$. Since the reproducing kernel $K_{w}=K(\cdot, \bar{w})$ at the point $w$ clearly belongs to $\mathcal{M}^{\perp}$ and $K_{w} \neq 0$ (since $K(w, \bar{w})>0$, see the beginning of the preceding proof), it follows that $\operatorname{dim} \mathcal{M}^{\perp}=1$.

Of course, the last theorem is of little practical use for such applications as in Theorem 1.3. In the following sections, we therefore establish the existence of $\frac{1}{K}$-calculi directly by construction in two interesting cases: for the standard weighted Bergman spaces on bounded symmetric domains, and for spaces $\mathcal{H}$ whose reproducing kernel is a rational function.

Note that none of the proofs in this section actually used the fact that the elements of $\mathcal{H}$ be holomorphic functions. Thus, all the results above extend to any functional Hilbert space (i.e., a Hilbert space whose elements are functions - no analyticity, or boundedness of point evaluations, etc., are assumed) on a set (not necessarily open) $\Omega \subset \mathbb{C}^{d}$. (In Proposition 1.5 and Theorem 1.6 we must assume that $K(z, \bar{w})$ is jointly continuous - implying, in particular, that the elements of $\mathcal{H}$ are continuous functions - and that $\{(z, w): K(z, \bar{w}) \neq 0\}$ is dense in $\Omega \times \Omega$.)

\section{BOUNDED SYMMETRIC DOMAINS}

Throughout this and the next section, let $\Omega$ be an irreducible bounded symmetric domain in $\mathbb{C}^{d}$ in its Harish-Chandra realization (i.e., a Cartan domain); thus $\Omega$ is circular, centered at the origin, and convex. We denote by $r, a, b, d$ and $p$ the rank, the characteristic multiplicities, the dimension and the genus of $\Omega$, respectively; these quantities are related by

$$
d=\frac{r(r-1)}{2} a+r b+r, \quad p=(r-1) a+b+2 .
$$

Let further $d m$ be the Lebesgue measure on $\mathbb{C}^{d}, G$ the identity connected component of the group of biholomorphic self-maps of $\Omega$, and $K$ the stabilizer of the origin in $G$. (There is little danger of confusion with the notation $K$ for the reproducing kernel.) The elements of $K$ are precisely the unitary maps on $\mathbb{C}^{d}$ preserving $\Omega$, and $G$ acts transitively on $\Omega \simeq G / K$. 
It is known that the reproducing kernel of the Bergman space $L_{\text {hol }}^{2}(\Omega, d m)$ comes in the form

$$
K(x, \bar{y})=c_{p} h(x, \bar{y})^{-p},
$$

where $c_{p}=m(\Omega)$ and the Jordan triple determinant $h(x, \bar{y})$ is an irreducible, $K$ invariant (i.e., $h(k x, \overline{k y})=h(x, \bar{y})$ for $k \in K)$ polynomial, nonvanishing on $\Omega \times \Omega$, holomorphic in $x$ and $\bar{y}$, and such that $h(x, 0)=h(0, \bar{x})=1 \forall x$. More generally, for each real $\nu>p-1$, there exists a constant $c_{\nu}>0$ such that

$$
d \mu_{\nu}(x):=c_{\nu} h(x, \bar{x})^{\nu-p} d m(x)
$$

is a probability measure, and the reproducing kernel of the weighted Bergman space

$$
\mathcal{H}_{\nu}:=L_{\text {hol }}^{2}\left(\Omega, d \mu_{\nu}\right)
$$

is given by $K_{\nu}(x, \bar{y})=h(x, \bar{y})^{-\nu}$. (For $\left.\nu \leq p-1, L_{\text {hol }}^{2}\left(\Omega, h^{\nu-p} d m\right)=\{0\}.\right)$

See e.g. Ara, $\mathrm{FK}$, or Lo] for more details.

Under the action $f \mapsto f \circ k(k \in K)$ of $K$, the space $\mathcal{P}$ of holomorphic polynomials on $\mathbb{C}^{d}$ admits the Peter-Weyl decomposition into a multiplicity-free direct sum of irreducible subspaces

$$
\mathcal{P}=\bigoplus_{\mathbf{m}} \mathcal{P}_{\mathbf{m}}
$$

where the summation extends over all signatures $\mathbf{m}$, i.e., $r$-tuples $\left(m_{1}, \ldots, m_{r}\right)$ of nonnegative integers such that $m_{1} \geq m_{2} \geq \cdots \geq m_{r} \geq 0$. For each $\mathbf{m}$, the elements of $\mathcal{P}_{\mathbf{m}}$ are homogeneous polynomials of degree $|\mathbf{m}|:=m_{1}+\cdots+m_{r}$; thus any holomorphic function on $\Omega$ has a Peter-Weyl expansion

$$
f=\sum_{\mathbf{m}} f_{\mathbf{m}}, \quad f_{\mathbf{m}} \in \mathcal{P}_{\mathbf{m}},
$$

which refines the familiar homogeneous expansion. The Fischer (or Fock) scalar product $\langle\cdot, \cdot\rangle_{F}$ on $\mathcal{P}$ is given by

$$
\begin{aligned}
\langle f, g\rangle_{F}: & =\left.f\left(\frac{\partial}{\partial z}\right) \overline{g(\bar{z})}\right|_{z=0} \\
& =\pi^{-d} \int_{\mathbb{C}^{d}} f(x) \overline{g(x)} e^{-|x|^{2}} d m(x) .
\end{aligned}
$$

One has $\langle f, g\rangle_{F}=0$ if $f \in \mathcal{P}_{\mathbf{m}}, g \in \mathcal{P}_{\mathbf{n}}$ with $\mathbf{m} \neq \mathbf{n}$. Conversely, it is known that for any $K$-invariant scalar product $\langle\langle\cdot, \cdot\rangle\rangle$ on $\mathcal{P}$, any two different spaces $\mathcal{P}_{\mathbf{m}}$ are orthogonal with respect to $\langle\langle\cdot, \cdot\rangle\rangle$, while on each $\mathcal{P}_{\mathbf{m}},\langle\langle\cdot, \cdot\rangle\rangle$ is proportional to $\langle\cdot, \cdot\rangle_{F} ;$ that is,

$$
\left\langle\left\langle\Sigma_{\mathbf{m}} f_{\mathbf{m}}, \Sigma_{\mathbf{m}} g_{\mathbf{m}}\right\rangle\right\rangle=\Sigma_{\mathbf{m}} \gamma_{\mathbf{m}}\left\langle f_{\mathbf{m}}, g_{\mathbf{m}}\right\rangle_{F}
$$

for certain constants $\gamma_{\mathbf{m}} \geq 0$. In particular, for the scalar products in $\mathcal{H}_{\nu}, \nu>p-1$, this holds with

$$
\gamma_{\mathbf{m}}=1 /(\nu)_{\mathbf{m}},
$$

where $(\nu)_{\mathbf{m}}$ is the generalized Pochhammer symbol

$$
(\nu)_{\mathbf{m}}:=\prod_{j=1}^{r}\left(\nu-\frac{j-1}{2} a\right)_{m_{j}}, \quad(\lambda)_{k}:=\lambda(\lambda+1) \ldots(\lambda+k-1) .
$$

The Hilbert spaces $\left(\mathcal{P}_{\mathbf{m}},\langle\cdot, \cdot\rangle_{F}\right)$ being finite-dimensional, the evaluation functionals at any $x \in \mathbb{C}^{d}$ are automatically continuous on them, and thus they have 
reproducing kernels $K_{\mathbf{m}}(x, \bar{y})$. For any orthonormal basis $\left\{\psi_{j}\right\}$ of $\mathcal{P}_{\mathbf{m}}$ (with respect to the Fock norm), these kernels satisfy (see $[\mathrm{Be}]$ )

$$
K_{\mathbf{m}}(x, \bar{y})=\sum_{j} \psi_{j}(x) \overline{\psi_{j}(y)}
$$

The following important formula, due to Faraut and Koranyi [FK], expresses the powers of the Jordan triple determinant $h$ in terms of $K_{\mathbf{m}}$ :

$$
h(x, \bar{y})^{-\nu}=\sum_{\mathbf{m}}(\nu)_{\mathbf{m}} K_{\mathbf{m}}(x, \bar{y}), \quad \forall \nu \in \mathbb{C},
$$

the convergence being uniform for $x, y$ in compact subsets of $\Omega$.

A simple consequence of the Faraut-Koranyi formula is that the function

$$
K_{\nu}(x, \bar{y})=h(x, \bar{y})^{-\nu}
$$

is positive definite not only for $\nu>p-1$, but actually for (and only for) all $\nu$ in the Wallach set

$$
\mathcal{W}=\left(\frac{r-1}{2} a, \infty\right) \cup\left\{\frac{j-1}{2} a, j=1,2, \ldots, r\right\}=: \mathcal{W}_{c} \cup \mathcal{W}_{d} ;
$$

$\mathcal{W}_{c}$ and $\mathcal{W}_{d}$ are called the continuous and the discrete Wallach sets, respectively. For each $\nu \in \mathcal{W}$, there exists a Hilbert space $\mathcal{H}_{\nu}$ of holomorphic functions on $\Omega$ with reproducing kernel $K_{\nu}$ (cf. $\overline{\text { Aro }}$ ). For $\nu>p-1$, these are the spaces $\mathcal{H}_{\nu}=L_{\text {hol }}^{2}\left(\Omega, d \mu_{\nu}\right)$ above. For $p-1 \geq \nu \geq d / r, \mathcal{H}_{\nu}$ is the closure of $\mathcal{P}$ in $L^{2}\left(d \mu_{\nu}\right)$ for certain Borel measures $\mu_{\nu}$ supported on subsets of the topological boundary $\partial \Omega$ of $\Omega\left[\mathrm{RV}\right.$ ] (see also [ArU]); in particular, the value $\nu=\frac{d}{r}$ corresponds to the Hardy space of $\Omega$, the closure of $\mathcal{P}$ in the space $L^{2}\left(\mu_{d / r}\right)$ where $\mu_{d / r}$ is the unique $K$-invariant probability measure on the Shilov boundary $\partial_{0} \Omega$ of $\Omega$. For $\nu<\frac{d}{r}$, the spaces $\mathcal{H}_{\nu}$ cannot be realized as holomorphic subspaces of any $L^{2}$ space. (They can be realized as Besov-type spaces, cf. [ArU].) The polynomials $\mathcal{P}$ are contained in $\mathcal{H}_{\nu}$ (and are dense in it) if and only if $\nu>\frac{r-1}{2} a$, i.e., $\nu \in \mathcal{W}_{c}$; for $\nu=\frac{j-1}{2} a \in \mathcal{W}_{d}$, $\mathcal{H}_{\nu}$ contains only the polynomials in $\bigoplus_{m_{1} \geq \cdots \geq m_{j}=m_{j+1}=\cdots=m_{r}=0} \mathcal{P}_{\mathbf{m}}$ (and these are dense in it). Finally, the commuting operator tuple $Z$ of multiplications by the coordinate functions maps $\mathcal{H}_{\nu}$ (boundedly) into itself if and only if $\nu>\frac{r-1}{2} a$; this is a recent result of $\mathrm{G}$. Zhang and the first author [ArZ].

The Shilov boundary $\partial_{0} \Omega$ coincides with the set of all points in $\partial \Omega$ of maximal Euclidean distance from the origin. The group $K$ operates transitively on $\partial_{0} \Omega$, so that for any $e \in \partial_{0} \Omega$, which we fix now once and for all, $\partial_{0} \Omega=\{k e, k \in K\} \simeq K / L$, where $L$ is the stabilizer of $e$ in $K$. Each Peter-Weyl space $\mathcal{P}_{\mathbf{m}}$ contains a unique $L$-invariant polynomial $\phi_{\mathbf{m}}$ satisfying the normalization condition $\phi_{\mathbf{m}}(e)=1$. The polynomials $\phi_{\mathbf{m}}$ are related to the reproducing kernels $K_{\mathbf{m}}$ by the formula

$$
K_{\mathbf{m}}(x, \bar{e})=\frac{d_{\mathbf{m}}}{(d / r)_{\mathbf{m}}} \phi_{\mathbf{m}}(x)
$$

where $d_{\mathbf{m}}:=\operatorname{dim} \mathcal{P}_{\mathbf{m}}$.

The polynomials $\phi_{\mathbf{m}}$ have also a combinatorial interpretation in terms of the Jack symmetric polynomials $J_{\mathbf{m}}^{(\lambda)}$ with parameter $\lambda$, cf. [MD, Section 10 of Chapter VI], KS. Namely, there exist vectors $e_{1}, \ldots, e_{r} \in \mathbb{C}^{d}$ (a Jordan frame), orthogonal with respect to the Euclidean metric on $\mathbb{C}^{d}$, such that $e_{1}+\cdots+e_{r}=e$ and each $x \in \mathbb{C}^{d}$ 
has a polar decomposition

$$
x=k \sum_{j=1}^{r} t_{j} e_{j}, \quad k \in K, t_{1} \geq t_{2} \geq \cdots \geq t_{r} \geq 0
$$

(the numbers $t_{1}, \ldots, t_{r}$ - the singular numbers of $x$ - are determined uniquely, but $k$ need not be). Further, $x$ belongs to $\Omega, \partial \Omega$, or $\mathbb{C}^{d} \backslash \Omega$ if and only if $t_{1}<1$, $t_{1}=1$, or $t_{1} \geq 1$, respectively. Now one has

$$
\phi_{\mathbf{m}}(x)=j_{\mathbf{m}} J_{\mathbf{m}}^{(2 / a)}\left(t_{1}^{2}, \ldots, t_{r}^{2}\right),
$$

where $j_{\mathbf{m}}=1 / J_{\mathbf{m}}^{(2 / a)}(1, \ldots, 1)$ is a normalizing constant (for which an explicit formula is known). We will suppress the superscript $(2 / a)$ in the sequel.

For two signatures $\mathbf{m}, \mathbf{n}$, the so-called Pieri rules express the product $J_{\mathbf{m}} J_{\mathbf{n}}$ as a linear combination of the $J_{\mathbf{k}}$ :

$$
J_{\mathbf{m}} J_{\mathbf{n}}=\sum_{\mathbf{k}} C_{\mathbf{m n}}^{\mathbf{k}} J_{\mathbf{k}}
$$

The coefficients $C_{\mathbf{m n}}^{\mathbf{k}}\left(=C_{\mathbf{n m}}^{\mathbf{k}}\right)$ are nonzero only when

$$
|\mathbf{k}|=|\mathbf{m}|+|\mathbf{n}| \quad \text { and } \quad \mathbf{m} \subset \mathbf{k}, \mathbf{n} \subset \mathbf{k},
$$

where, for two signatures $\mathbf{m}$ and $\mathbf{k}, \mathbf{m} \subset \mathbf{k}$ means $m_{j} \leq k_{j} \forall j=1, \ldots, r$.

The numbers $C_{\mathbf{m n}}^{\mathbf{k}}$ have been computed for $\mathbf{n}=\left(1^{j}\right)$ and for $\mathbf{n}=(j)$ by Lassalle [La] and Stanley [St, Theorem 6.1], respectively (cf. [MD], Chapter VI, Sections 6 and 7, in particular (6.24) and (10.11); see also Zhang [Zh] and Vretare [Vr]). Explicit formulas and properties of these coefficients for general $\mathbf{m}, \mathbf{n}, \mathbf{k}$ are unknown (although in principle $C_{\mathbf{m n}}^{\mathbf{k}}$ are explicitly computable, see [MD p. 351]).

From $(2.7),(2.6)$ and (2.4) we immediately obtain also a multiplication formula for the reproducing kernels $K_{\mathrm{m}}$ :

$$
K_{\mathbf{m}}(x, \bar{y}) K_{\mathbf{n}}(x, \bar{y})=\sum_{\mathbf{k}} c_{\mathbf{m n}}^{\mathbf{k}} K_{\mathbf{k}}(x, \bar{y}),
$$

where the coefficients

$$
c_{\mathbf{m} \mathbf{n}}^{\mathbf{k}}=\frac{d_{\mathbf{m}} j_{\mathbf{m}} d_{\mathbf{n}} j_{\mathbf{n}}(d / r)_{\mathbf{k}}}{(d / r)_{\mathbf{m}}(d / r)_{\mathbf{n}} d_{\mathbf{k}} j_{\mathbf{k}}} C_{\mathbf{m n}}^{\mathbf{k}}
$$

are again nonzero only if $(2.8)$ holds.

Note that the coefficients $C_{\mathbf{m n}}^{\mathbf{k}}$ and $c_{\mathbf{m n}}^{\mathbf{k}}$, as well as the Jack polynomials themselves, depend only on $r$ and $a$, but not on $b$.

Finally, we will need the following formula for the dimensions $d_{\mathbf{m}}$ :

$$
d_{\mathbf{m}}=\frac{(d / r)_{\mathbf{m}}}{\left(d_{1} / r\right)_{\mathbf{m}}} \prod_{1 \leq i<j \leq r} \frac{m_{i}-m_{j}+\frac{j-i}{2} a}{\frac{j-i}{2} a} \frac{\left(\frac{j-i+1}{2} a\right)_{m_{i}-m_{j}}}{\left(\frac{j-i-1}{2} a+1\right)_{m_{i}-m_{j}}},
$$

where $d_{1}=d-r b=r(r-1) \frac{a}{2}+r$ is the dimension of the tube-type "subdomain"1 of $\Omega$. See [Up, Lemmas 2.5 and 2.6.

\footnotetext{
${ }^{1}$ It is not really a subdomain, but an intersection of $\Omega$ with a subspace of lower dimension.
} 


\section{3. $\frac{1}{K}$-CALCUli For CARTAN Domains}

We proceed to discuss the construction of $\frac{1}{K}$-calculi on Cartan domains. Recall that for a holomorphic polynomial $p(x, \bar{y})$ in $x, \bar{y}$ and a commuting operator tuple $T$, we have defined $p\left(T, T^{*}\right)$ by writing all $T$ 's to the left of all $T^{*}$ 's, that is,

$$
p\left(T, T^{*}\right):=p\left(L_{T}, R_{T^{*}}\right) I .
$$

Our starting point is the following proposition.

Proposition 3.1. Let $\mathcal{H}$ be a Hilbert space of holomorphic functions on a domain $\Omega \subset \mathbb{C}^{d}$ satisfying the conditions (1), (2) and (3). Assume that $K(x, \bar{y}) \neq 0 \forall x, y \in$ $\Omega$ and that there exists a sequence of polynomials $p_{k}(x, \bar{y})$ on $\Omega \times \Omega(k=1,2, \ldots)$, holomorphic in $x$ and $\bar{y}$, such that

$$
p_{k}(x, \bar{y}) \rightarrow \frac{1}{K(x, \bar{y})} \quad \text { as } k \rightarrow \infty, \forall x, y \in \Omega
$$

and

$$
\sup _{k}\left\|p_{k}\left(Z, Z^{*}\right)\right\|<\infty
$$

Then the recipe

$$
\begin{aligned}
& \operatorname{dom} \Phi_{H}:=\left\{T \in \mathcal{B}(\mathcal{H}): \sup _{k}\left\|p_{k}\left(T, T^{*}\right)\right\|<\infty,\right. \text { and the limit } \\
& \left.\quad \lim _{k \rightarrow \infty}^{(\mathrm{WOT})} p_{k}\left(T, T^{*}\right) \text { exists and is a nonnegative operator }\right\}, \\
& \Phi_{H}(T):=\lim _{k \rightarrow \infty}^{(\mathrm{WOT})} p_{k}\left(T, T^{*}\right), \\
& \text { defines a strong } \frac{1}{K} \text {-calculus for } \mathcal{H} .
\end{aligned}
$$

Here $\lim ^{(\text {WOT })}$ stands for the limit in the weak operator topology.

Proof. Using (1.2) we see that, for $K_{w}:=K(\cdot, \bar{w})$,

$$
\left\langle p_{k}\left(Z, Z^{*}\right) K_{y}, K_{x}\right\rangle \rightarrow 1=\left\langle P_{0} K_{y}, K_{x}\right\rangle \quad \forall x, y \in \Omega
$$

(cf. the proof of Proposition 1.2). Since the linear span of $\left\{K_{w}\right\}_{w \in \Omega}$ is dense in $\mathcal{H}$, (3.2) implies that $p_{k}\left(Z, Z^{*}\right) \rightarrow P_{0}$ in the weak operator topology. Consequently, for any separable Hilbert space $E$ and $M=Z \otimes I_{E}$, we have $\sup _{k}\left\|p_{k}\left(M, M^{*}\right)\right\|=$ $\sup _{k}\left\|p_{k}\left(Z, Z^{*}\right)\right\|<\infty$ and $\lim _{k \rightarrow \infty}^{(\mathrm{WOT})} p_{k}\left(M, M^{*}\right)$ exists and equals $P_{0} \otimes I_{E}$, a nonnegative operator. Thus $M \in \operatorname{dom} \Phi_{H}$ and the condition $(\beta)$ in Definition 1.1 is satisfied. If $V: H_{1} \rightarrow H_{2}$ is an isometry and $T_{1} V^{*}=V^{*} T_{2}$, then for any multiindices $\alpha$ and $\beta$,

$$
T_{1}^{\alpha} T_{1}^{* \beta}=T_{1}^{\alpha} V^{*} V T_{1}^{* \beta}=V^{*} T_{2}^{\alpha} T_{2}^{* \beta} V
$$

hence $p\left(T_{1}, T_{1}^{*}\right)=V^{*} p\left(T_{2}, T_{2}^{*}\right) V$ for any $p$. Thus $T_{2} \in \operatorname{dom} \Phi_{H_{2}}$ implies that $T_{1} \in \operatorname{dom} \Phi_{H_{1}}$ and

$$
\Phi_{H_{1}}\left(T_{1}\right)=\lim _{k \rightarrow \infty}^{(\mathrm{WOT})} V^{*} p_{k}\left(T_{2}, T_{2}^{*}\right) V=V^{*} \Phi_{H_{2}}\left(T_{2}\right) V .
$$

So the condition $(\alpha)$ of Definition 1.1 is satisfied as well.

Recall that for any commuting operator tuple $T \subset \mathcal{B}(\mathcal{H})$, nonnegative operator $C$ on $H$, and orthonormal basis $\boldsymbol{\psi}$ of $\mathcal{H}$, the sequence of operators $f_{m, C}(T)=$ $f_{m, C, \psi}(T) \in \mathcal{B}(\mathcal{H})$ was defined by $(1.3)$.

Corollary 3.2. Under the hypotheses of Proposition 3.1, the following assertions about a commuting operator tuple $T$ on a separable Hilbert space $H$ are equivalent: 
(a) $T^{*}$ is unitarily equivalent to the restriction to an invariant subspace of the direct sum of a countable number of copies of $Z^{*}$ (i.e., of $Z^{*} \otimes I_{E}$ for some auxiliary separable Hilbert space E);

(b) $\sup _{k}\left\|p_{k}\left(T, T^{*}\right)\right\|<\infty$, the limit $\lim _{k \rightarrow \infty}^{(\mathrm{WOT})} p_{k}\left(T, T^{*}\right)=$ : $C$ exists and is nonnegative, and $f_{m, C}(T) \rightarrow 0$ strongly as $m \rightarrow \infty$.

Let us now turn to the Cartan domains $\Omega$ and the (analytic continuation of the) weighted Bergman spaces $\mathcal{H}_{\nu}$ on them, described in the preceding section. From the formula $K_{\nu}(x, \bar{y})=h(x, \bar{y})^{-\nu}$, it is immediate that the reproducing kernels $K_{\nu}$ do not vanish anywhere on $\Omega \times \Omega$. Also, we have seen that $\mathcal{H}_{\nu}$ satisfies the conditions (1) and (3) if and only if $\nu>\frac{r-1}{2} a$. Note that $\frac{d}{r}=\frac{r-1}{2} a+b+1>\frac{r-1}{2} a$.

Theorem 3.3. Let $\nu \geq d / r$. Then the polynomials

$$
p_{k}(x, \bar{y})=\sum_{|\mathbf{m}| \leq k}(-\nu)_{\mathbf{m}} K_{\mathbf{m}}(x, \bar{y})
$$

satisfy the conditions (3.1) and (3.2).

Proof. By the Faraut-Koranyi formula, we have $p_{k}(x, \bar{y}) \rightarrow h(x, \bar{y})^{\nu}=1 / K_{\nu}(x, \bar{y})$ uniformly on compact subsets of $\Omega \times \Omega$; this settles (3.1). To prove (3.2), it clearly suffices to show that

$$
\sum_{\mathbf{m}}\left|(-\nu)_{\mathbf{m}}\right|\left\|K_{\mathbf{m}}\left(Z, Z^{*}\right)\right\|<\infty .
$$

This, in turn, is immediate from the following two propositions and (2.4).

Proposition 3.4. For $\nu \geq d / r$,

$$
\left\|K_{\mathbf{m}}\left(Z, Z^{*}\right)\right\| \leq K_{\mathbf{m}}(e, \bar{e}) .
$$

Proof. Pick any orthonormal basis $\left\{\psi_{j}\right\}$ of $\mathcal{P}_{\mathbf{m}}$ (with respect to the Fock norm). By (2.3),

$$
K_{\mathbf{m}}\left(Z, Z^{*}\right)=\sum_{j} \psi_{j}(Z) \psi_{j}(Z)^{*}=\mathbf{M M}^{*},
$$

where $\mathbf{M}$ is the row block operator $\mathbf{M}=\left[\psi_{1}(Z), \ldots, \psi_{d_{\mathbf{m}}}(Z)\right]: \mathcal{H}_{\nu}^{\oplus d_{\mathbf{m}}} \rightarrow \mathcal{H}_{\nu}$. Now by the result of Rossi and Vergne, for $\nu \geq d / r$ the space $\mathcal{H}_{\nu}$ is the holomorphic subspace of some $L^{2}$ space (i.e., the closure of holomorphic polynomials in $L^{2}\left(d \mu_{\nu}\right)$ for some measure $\mu_{\nu}$ supported in $\left.\bar{\Omega}\right)$; consequently, for any $\mathbf{f}=\left[f_{1}, \ldots, f_{d_{\mathbf{m}}}\right]^{t} \in \mathcal{H}_{\nu}^{\oplus d_{\mathbf{m}}}$,

$$
\begin{aligned}
\|\mathbf{M f}\|^{2} & =\left\|\sum_{j} \psi_{j} f_{j}\right\|^{2}=\int\left|\sum_{j} \psi_{j} f_{j}\right|^{2} d \mu_{\nu} \\
& \leq \int\left(\sum\left|\psi_{j}\right|^{2}\right)\left(\sum\left|f_{j}\right|^{2}\right) d \mu_{\nu} \\
& \leq \operatorname{ess}-\sup \left(\sum\left|\psi_{j}\right|^{2}\right) \cdot \int\left(\sum\left|f_{j}\right|^{2}\right) d \mu_{\nu} \\
& \leq \operatorname{ess-sup}\left(\sum\left|\psi_{j}\right|^{2}\right) \cdot\|\mathbf{f}\|^{2} .
\end{aligned}
$$


Thus

$$
\begin{aligned}
\left\|\mathbf{M M}^{*}\right\|=\|\mathbf{M}\|^{2} & \leq \sup _{\bar{\Omega}} \sum\left|\psi_{j}\right|^{2} \\
& =\sup _{x \in \bar{\Omega}} K_{\mathbf{m}}(x, \bar{x}) \\
& =K_{\mathbf{m}}(e, \bar{e}) \quad(\text { see }[\underline{\mathrm{FK}}, \text { p. } 78),
\end{aligned}
$$

as claimed.

Proposition 3.5. The series

$$
\sum_{\mathbf{m}}\left|(-\nu)_{\mathbf{m}}\right| \frac{d_{\mathbf{m}}}{(d / r)_{\mathbf{m}}}
$$

converges if and only if $\nu>\frac{r-1}{2} a$.

Proof. For two functions $f(\mathbf{m})$ and $g(\mathbf{m})$ on the set of all partitions, we will write $f \lesssim g$ if

$$
f(\mathbf{m}) \leq C g(\mathbf{m}) \quad \forall \mathbf{m}
$$

for some finite positive constant $C$ independent of $\mathbf{m}$, and $f \asymp g$ if $f \lesssim g$ and $g \lesssim f$. From the definition of the generalized Pochhammer symbol we then get

$$
\frac{\left|(-\nu)_{\mathbf{m}}\right|}{(d / r)_{\mathbf{m}}} \asymp \prod_{j=1}^{r}\left(1+m_{j}\right)^{-\nu-d / r} .
$$

Similarly, using (2.11) it is not difficult to see that

$$
d_{\mathbf{m}} \asymp \prod_{1 \leq i<j \leq r}\left(1+m_{i}-m_{j}\right)^{a} \cdot \prod_{j=1}^{r}\left(1+m_{j}\right)^{b} .
$$

Thus on the one hand,

$$
\begin{aligned}
\sum_{\mathbf{m}}\left|(-\nu)_{\mathbf{m}}\right| \frac{d_{\mathbf{m}}}{(d / r)_{\mathbf{m}}} & \asymp \sum_{\mathbf{m}}\left(\prod_{j=1}^{r}\left(1+m_{j}\right)^{b-\nu-d / r} \cdot \prod_{1 \leq i<j \leq r}\left(1+m_{i}-m_{j}\right)^{a}\right) \\
& \leq \sum_{\mathbf{m}}\left(\prod_{j=1}^{r}\left(1+m_{j}\right)^{b-\nu-d / r} \cdot \prod_{1 \leq i \leq r}\left(1+m_{i}\right)^{(r-1) a}\right) \\
& \leq \sum_{m_{1}, \ldots, m_{r}=0}^{\infty} \prod_{j=1}^{r}\left(1+m_{j}\right)^{(r-1) a+b-\nu-d / r} \\
& =\left[\sum_{m=0}^{\infty}(1+m)^{(r-1) a+b-\nu-d / r}\right]^{r},
\end{aligned}
$$


while on the other hand,

$$
\begin{aligned}
\sum_{\mathbf{m}} \frac{\left|(-\nu)_{\mathbf{m}}\right| d_{\mathbf{m}}}{(d / r)_{\mathbf{m}}} & \gtrsim \sum_{\substack{m_{1} \geq 0, \mathbf{m}=\left(m_{1}, 0,0, \ldots, 0\right)}}\left(\prod_{j=1}^{r}\left(1+m_{j}\right)^{b-\nu-d / r} \cdot \prod_{1 \leq i<j \leq r}\left(1+m_{i}-m_{j}\right)^{a}\right) \\
& =\sum_{m_{1}=0}^{\infty}\left(1+m_{1}\right)^{b-\nu-d / r} \prod_{1<j \leq r}\left(1+m_{1}\right)^{a} \\
& =\sum_{m_{1}=0}^{\infty}\left(1+m_{1}\right)^{(r-1) a+b-\nu-d / r},
\end{aligned}
$$

i.e., the same expression as for the upper bound except for the missing $r$-th power. Thus the series in question converges if and only if

$$
\sum_{k=1}^{\infty} k^{(r-1) a+b-\nu-d / r}<\infty,
$$

which happens if and only if $(r-1) a+b-\nu-d / r<-1$, i.e., if and only if $\nu>\frac{r-1}{2} a$.

The fact that we did not use the full force of Proposition 3.5 in the proof of the last theorem suggests that the latter might, in fact, hold also for some $\nu$ in the interval between $\frac{d}{r}$ and $\frac{r-1}{2} a$. We are going to show that this is indeed the case at least for $\Omega$ the unit ball in $\mathbb{C}^{d}$. We start by establishing several general facts about the operators $K_{\mathbf{m}}\left(Z, Z^{*}\right)$.

From the $K$-invariance of $K_{\mathbf{m}}(x, \bar{y})$ and the Schur lemma, it follows that the operators $K_{\mathbf{m}}\left(Z, Z^{*}\right)$ are diagonal with respect to the Peter-Weyl decomposition (2.1); that is,

$$
\left.K_{\mathbf{k}}\left(Z, Z^{*}\right)\right|_{\mathcal{P}_{\mathbf{m}}}=\tau_{\mathbf{k}}(\mathbf{m}) I_{\mathcal{P}_{\mathbf{m}}}, \quad \forall \mathbf{m}, \mathbf{k}
$$

for some complex numbers $\tau_{\mathbf{k}}(\mathbf{m})$; since $K_{\mathbf{m}}\left(Z, Z^{*}\right) \geq 0$ (cf. (3.5)), these numbers are in fact nonnegative. The next proposition shows that they can be expressed in terms of the Pieri coefficients (2.10).

Proposition 3.6. The following formula holds:

$$
\tau_{\mathbf{k}}(\mathbf{m})=\sum_{\mathbf{j}} \frac{(\nu)_{\mathbf{j}}}{(\nu)_{\mathbf{m}}} c_{\mathbf{k j}}^{\mathbf{m}}
$$

(Observe that in view of (2.8), the last sum contains only finitely many nonzero terms.) In particular, $\tau_{\mathbf{k}}(\mathbf{m})$ is a rational function of $\nu$ of degree $|\mathbf{k}|$, with poles (possibly) only at the points $\nu=\frac{s-1}{2} a-t \in\left(-\infty, \frac{r-1}{2} a\right], s=1, \ldots, r, t=$ $j_{s}, j_{s}+1, \ldots, m_{s}-1$.

Remark. As a corollary to the last proposition, we see that $Z_{1} Z_{1}^{*}+\cdots+Z_{d} Z_{d}^{*}=$ $K_{(1,0, \ldots, 0)}\left(Z, Z^{*}\right)$ is bounded for $\nu>\frac{r-1}{2} a$, hence so are $Z_{1}, \ldots, Z_{d}$. This is part of the result of Zhang and the first author in [ArZ]. The proof below uses the same idea as the proof of Theorem 4.5 there.

Proof of Proposition 3.6. Let $f_{\mathbf{m}} \in \mathcal{P}_{\mathbf{m}}$. By the reproducing property of the kernel and the selfadjointness of $K_{\mathbf{k}}\left(Z, Z^{*}\right)$, we have

$$
\begin{aligned}
\tau_{\mathbf{k}}(\mathbf{m}) f_{\mathbf{m}}(w) & =\left\langle K_{\mathbf{k}}\left(Z, Z^{*}\right) f_{\mathbf{m}}, K(\cdot, \bar{w})\right\rangle_{\nu} \\
& =\left\langle f_{\mathbf{m}}, K_{\mathbf{k}}\left(Z, Z^{*}\right) K(\cdot, \bar{w})\right\rangle_{\nu} .
\end{aligned}
$$


By (1.2), the Faraut-Koranyi formula, and (2.9),

$$
\begin{aligned}
K_{\mathbf{k}}\left(Z, Z^{*}\right) K(\cdot, \bar{w}) & =K_{\mathbf{k}}(\cdot, \bar{w}) K(\cdot, \bar{w}) \\
& =\sum_{\mathbf{j}} K_{\mathbf{k}}(\cdot, \bar{w})(\nu)_{\mathbf{j}} K_{\mathbf{j}}(\cdot, \bar{w}) \\
& =\sum_{\mathbf{j}, \mathbf{n}}(\nu)_{\mathbf{j}} c_{\mathbf{k j}}^{\mathbf{n}} K_{\mathbf{n}}(\cdot, \bar{w}) .
\end{aligned}
$$

Since, by the orthogonality of $\mathcal{P}_{\mathbf{m}}$ and the Faraut-Koranyi formula,

we thus get

$$
\left\langle f_{\mathbf{m}},(\nu)_{\mathbf{n}} K_{\mathbf{n}}(\cdot, \bar{w})\right\rangle_{\nu}=\left\{\begin{array}{lll}
f_{\mathbf{n}}(w) & \text { if } & \mathbf{m}=\mathbf{n}, \\
0 & \text { if } & \mathbf{m} \neq \mathbf{n},
\end{array}\right.
$$

$$
\begin{aligned}
\tau_{\mathbf{k}}(\mathbf{m}) f_{\mathbf{m}}(w) & =\sum_{\mathbf{j}, \mathbf{n}}\left\langle f_{\mathbf{m}},(\nu)_{\mathbf{n}} K_{\mathbf{n}}(\cdot, \bar{w})\right\rangle_{\nu} \frac{(\nu)_{\mathbf{j}}}{(\nu)_{\mathbf{n}}} c_{\mathbf{k j}}^{\mathbf{n}} \\
& =\left(\sum_{\mathbf{j}} \frac{(\nu)_{\mathbf{j}}}{(\nu)_{\mathbf{m}}} c_{\mathbf{k j}}^{\mathbf{m}}\right) f_{\mathbf{m}}(w),
\end{aligned}
$$

and the formula (3.6) follows. The rest is immediate from the fact that for $\mathbf{j} \not \subset \mathbf{~ m}$ the coefficient $c_{\mathbf{k j}}^{\mathbf{m}}$ is zero, while if $\mathbf{j} \subset \mathbf{m}$, then $\frac{(\nu)_{\mathbf{m}}}{(\nu)_{\mathbf{j}}}$ is a monic polynomial in $\nu$ of degree $|\mathbf{m}|-|\mathbf{j}|=|\mathbf{k}|$ of the form $\prod\left(\nu+t-\frac{s-1}{2} a\right)$, with $s$ and $t$ as indicated in the proposition.

We also record the following simple observation.

Proposition 3.7. For all signatures $\mathbf{m}$ and $\nu_{1}, \nu_{2} \in \mathbb{C}$,

$$
\sum_{\mathbf{k}, \mathbf{j}} c_{\mathbf{k j}}^{\mathbf{m}}\left(\nu_{1}\right)_{\mathbf{k}}\left(\nu_{2}\right)_{\mathbf{j}}=\left(\nu_{1}+\nu_{2}\right)_{\mathbf{m}} .
$$

Proof. Apply the Faraut-Koranyi formula on both sides of the equality

$$
h^{-\nu_{1}} \cdot h^{-\nu_{2}}=h^{-\nu_{1}-\nu_{2}},
$$

use (2.9), and compare the coefficients at $K_{\mathbf{m}}$.

The following corollary gives a precise meaning to the equality $\frac{1}{K}\left(Z, Z^{*}\right)=P_{0}$ (that is, $h^{\nu}\left(L_{Z}, R_{Z^{*}}\right) I=P_{0}$ ) even for $\frac{1}{K}$ not a polynomial (i.e., $\nu$ not an integer).

Corollary 3.8. $\sum_{\mathbf{k}}(-\nu)_{\mathbf{k}} \tau_{\mathbf{k}}(\mathbf{m})=1$ if $\mathbf{m}=(0)$, and $=0$ otherwise.

Proof. Use (3.6) and apply the last proposition with $\nu_{2}=\nu=-\nu_{1}$.

Let us now apply the above facts to the case of the unit ball.

Proposition 3.9. Let $\Omega=\mathbb{B}^{d}$ be the unit ball in $\mathbb{C}^{d}$. Then

$$
\sum_{\mathbf{m}}\left|(-\nu)_{\mathbf{m}}\right|\left\|K_{\mathbf{m}}\left(Z, Z^{*}\right)\right\|<\infty \Longleftrightarrow \nu>\frac{1}{2}
$$

and

$$
\sup _{k}\left\|\sum_{|\mathbf{m}| \leq k}\left|(-\nu)_{\mathbf{m}}\right| K_{\mathbf{m}}\left(Z, Z^{*}\right)\right\|<\infty \quad \text { for all } \nu>0 .
$$

In particular, the conclusion of Corollary 3.2, with $p_{k}$ the polynomials given by (3.3), holds for all $\nu>0$. 
Proof. For the ball we have $r=1$; so $\mathbf{m}=(m)$, and $\phi_{m}=\phi_{1}^{m}$, whence

$$
c_{m n}^{k}=\left\{\begin{array}{cl}
\left(\begin{array}{c}
k \\
m
\end{array}\right) & \text { if } k=m+n, \\
0 & \text { otherwise. }
\end{array}\right.
$$

Consequently, by (3.6),

$$
\tau_{l}(m)=\left\{\begin{array}{l}
\frac{(\nu)_{m-l}}{(\nu)_{m}}\left(\begin{array}{c}
m \\
l
\end{array}\right)=\frac{1}{l !} \prod_{j=m-l}^{m-1} \frac{j+1}{j+\nu} \quad \text { if } \quad m \geq l, \\
0 \quad \text { if } \quad m<l .
\end{array}\right.
$$

Observe that $\frac{j+1}{j+\nu}$ increases with $j$ if $\nu>1$, but decreases with increasing $j$ if $\nu<1$. Thus,

$$
\left\|K_{l}\left(Z, Z^{*}\right)\right\|=\sup _{m} \tau_{l}(m)=\left\{\begin{array}{l}
\lim _{m \rightarrow \infty} \tau_{l}(m)=1 / l ! \quad \text { if } \quad \nu \geq 1, \\
\tau_{l}(l)=1 /(\nu)_{l} \quad \text { if } \quad \nu \leq 1 .
\end{array}\right.
$$

Consequently, for $\nu \geq 1$,

$$
\sum_{l=0}^{\infty}\left|(-\nu)_{l}\right|\left\|K_{l}\left(Z, Z^{*}\right)\right\|=\sum_{l=0}^{\infty}\left|\frac{(-\nu)_{l}}{(1)_{l}}\right| \sim \sum_{l=0}^{\infty}(1+l)^{-\nu-1}<\infty,
$$

while for $0<\nu \leq 1$,

$$
\sum_{l=0}^{\infty}\left|(-\nu)_{l}\right||| K_{l}\left(Z, Z^{*}\right) \|=\sum_{l=0}^{\infty}\left|\frac{(-\nu)_{l}}{(\nu)_{l}}\right| \sim \sum_{l=0}^{\infty}(1+l)^{-2 \nu},
$$

which converges if and only if $\nu>\frac{1}{2}$. This completes the proof of (3.7).

For (3.8), it suffices to consider only $\nu$ in the interval $0<\nu \leq \frac{1}{2}$. However, in that case, $(-\nu)_{0}=1$ and

$$
\left|(-\nu)_{j}\right|=-(-\nu)_{j} \quad \text { if } \quad j \geq 1 .
$$

It follows that

$$
\begin{aligned}
\left\|\sum_{l=0}^{k}\left|(-\nu)_{l}\right| K_{l}\left(Z, Z^{*}\right)\right\| & =\sup _{m}\left|\sum_{l=0}^{k}\right|(-\nu)_{l}\left|\tau_{l}(m)\right| \\
& =\sup _{m}\left[\tau_{0}(m)-\sum_{l=1}^{k}(-\nu)_{l} \tau_{l}(m)\right] \\
& \leq \sup _{m}\left[\tau_{0}(m)-\sum_{l=1}^{\infty}(-\nu)_{l} \tau_{l}(m)\right] \\
& =\sup _{m}\left[2 \tau_{0}(m)-\sum_{l=0}^{\infty}(-\nu)_{l} \tau_{l}(m)\right] \\
& =\sup _{m}\left[2-\delta_{0 m}\right] \text { by Corollary } 3.8 \\
& =2<\infty,
\end{aligned}
$$

which establishes the claim.

The results above suggest the following two conjectures, whose proofs hinge upon a better understanding of the Pieri coefficients $c_{\mathbf{m n}}^{\mathbf{k}}$. 
Conjecture A. For any Cartan domain,

$$
\left\|K_{\mathbf{m}}\left(Z, Z^{*}\right)\right\| \asymp \begin{cases}1 /\left(d_{1} / r\right)_{\mathbf{m}} & \text { if } \nu \geq d_{1} / r, \\ 1 /(\nu)_{\mathbf{m}} & \text { if } \nu \leq d_{1} / r .\end{cases}
$$

Conjecture B. For any Cartan domain and any $\nu>\frac{r-1}{2} a$,

$$
\sup _{\mathbf{m}}\left[\sum_{\mathbf{k}}\left|(-\nu)_{\mathbf{k}}\right| \tau_{\mathbf{k}}(\mathbf{m})\right]<\infty
$$

\section{RATIONAL REPRODUCING KERNELS}

In this section we consider Hilbert spaces $\mathcal{H}$ of holomorphic functions on domains $\Omega \subset \mathbb{C}^{d}$ whose reproducing kernels are rational functions. That is, we will assume that

there exist holomorphic polynomials $P, Q$ on $\mathbb{C}^{d} \times \mathbb{C}^{d}$ without a common factor such that $P$ is not identically zero and the reproducing kernel of

$$
K(x, \bar{y})=\frac{P(x, \bar{y})}{Q(x, \bar{y})}
$$

Observe that (4.1) implies, in particular, that

$$
Q(x, \bar{y}) \neq 0 \quad \forall x, y \in \Omega .
$$

We would now like to construct a $\frac{1}{K}$-calculus for $\mathcal{H}$, that is, to define, for $T$ a commuting operator tuple on a separable Hilbert space $H$,

$$
P\left(L_{T}, R_{T^{*}}\right)^{-1} Q\left(L_{T}, R_{T^{*}}\right) I .
$$

The obvious problem is that $P\left(L_{T}, R_{T^{*}}\right)$ need not be an invertible element of $\mathcal{B}(\mathcal{B}(\mathcal{H}))$, even for the "prototype" operator tuple $T=Z$.

Example 4.1. Let $\Omega=\mathbb{D}$ and let $\mathcal{H}$ be the Hilbert space of all analytic functions $f(z)=\sum_{0}^{\infty} f_{j} z^{j}$ on $\mathbb{D}$ satisfying

$$
\|f\|_{\mathcal{H}}^{2}:=\left|f_{0}\right|^{2}+\frac{1}{3} \sum_{j=1}^{\infty}\left|f_{j}\right|^{2}<\infty .
$$

(As a set, $\mathcal{H}$ coincides with the Hardy space $H^{2}$.) Then

$$
K(x, \bar{y})=1+\sum_{j=1}^{\infty} 3 x^{j} \bar{y}^{j}=\frac{1+2 x \bar{y}}{1-x \bar{y}},
$$

which is of the form (4.1) with $Q(x, \bar{y})=1-x \bar{y}$ and $P(x, \bar{y})=1+2 x \bar{y}$. Now by general results of Curto $[\mathrm{Cu}$, for any holomorphic polynomial $p$ in two variables and commuting operator tuple $T$, the spectrum of $p\left(L_{T}, R_{T^{*}}\right)$ is given by

$$
\sigma\left(p\left(L_{T}, R_{T^{*}}\right)\right)=\left\{p(\alpha, \bar{\beta}): \alpha, \beta \in \sigma_{\text {Taylor }}(T)\right\} .
$$

Consequently, in our case,

$$
\sigma\left(P\left(L_{Z}, R_{Z^{*}}\right)\right)=\{1+2 \alpha \bar{\beta}: \alpha, \beta \in \overline{\mathbb{D}}\}=1+2 \overline{\mathbb{D}} \ni 0 ;
$$

so $P\left(L_{Z}, R_{Z^{*}}\right)$ is not invertible.

Nonetheless, it turns out that it is still possible to define (4.3) consistently for $T=Z$, and use this to construct a $\frac{1}{K}$-calculus for $\mathcal{H}$. 
Proposition 4.2. Let $\Omega$ and $\mathcal{H}$ satisfy (4.1). Then the equation

$$
P\left(L_{Z}, R_{Z^{*}}\right) C=Q\left(L_{Z}, R_{Z^{*}}\right) I
$$

has the unique solution $C=P_{0}$.

Proof. Let $X \in \mathcal{B}(\mathcal{H})$ satisfy

$$
P\left(L_{Z}, R_{Z^{*}}\right) X=0
$$

Then for any $z, w \in \Omega$,

$$
0=\left\langle P\left(L_{Z}, R_{Z^{*}}\right) X K_{w}, K_{z}\right\rangle=P(z, \bar{w})\left\langle X K_{w}, K_{z}\right\rangle
$$

(here, as before, $K_{w}:=K(\cdot, \bar{w})$ ). It follows that $\left\langle X K_{w}, K_{z}\right\rangle=0$ almost everywhere on $\Omega \times \Omega$. Since $\left\langle X K_{w}, K_{z}\right\rangle$ is holomorphic in $z$ and $\bar{w}$ and the linear span of $\left\{K_{w}\right\}_{w \in \Omega}$ is dense in $\mathcal{H}$, this implies that $X=0$. Thus $P\left(L_{Z}, R_{Z^{*}}\right)$ is injective, which proves the uniqueness of $C$.

On the other hand, for $C=P_{0}$ and any $z, w \in \Omega$,

$$
\left\langle P\left(L_{Z}, R_{Z^{*}}\right) C K_{w}, K_{z}\right\rangle=P(z, \bar{w})\left\langle C K_{w}, K_{z}\right\rangle=P(z, \bar{w}) \overline{\mathbf{1}(w)} \mathbf{1}(z)=P(z, \bar{w}),
$$

while

$$
\left\langle Q\left(L_{Z}, R_{Z^{*}}\right) I K_{w}, K_{z}\right\rangle=Q(z, \bar{w})\left\langle I K_{w}, K_{z}\right\rangle=Q(z, \bar{w}) K(z, \bar{w}),
$$

which equals $P(z, \bar{w})$ in view of (4.1).

Theorem 4.3. Let $\Omega$ and $\mathcal{H}$ satisfy (4.1). Then the recipe

$$
\begin{aligned}
& \operatorname{dom} \Phi_{H}:=\left\{T \in \mathcal{B}(\mathcal{H}): \exists C \in \mathcal{B}(\mathcal{H})^{+} \text {such that } P\left(L_{T}, R_{T^{*}}\right) C=Q\left(L_{T}, R_{T^{*}}\right) I\right\}, \\
& \Phi_{H}(T):=\text { the set of all such } C, \\
& \text { defines a } \frac{1}{K} \text {-calculus for } \mathcal{H} \text {. }
\end{aligned}
$$

Proof. We have to check that the conditions $(\alpha)$ and $(\beta)$ from Definition 1.1 are satisfied. The validity of $(\beta)$ follows immediately from the last proposition. To prove $(\alpha)$ let $V: H_{1} \rightarrow H_{2}$ be an isometry such that $T_{1} V^{*}=V^{*} T_{2}$ and let $T_{2} \in \operatorname{dom} \Phi_{H_{2}}$. For any $C_{2} \in \Phi_{H_{2}}\left(T_{2}\right)$, the relations

$$
L_{T_{1}} L_{V^{*}}=L_{V^{*}} L_{T_{2}}, \quad R_{T_{1}^{*}} R_{V}=R_{V} R_{T_{2}^{*}}
$$

then imply

$$
\begin{aligned}
P\left(L_{T_{1}}, R_{T_{1}^{*}}\right) L_{V^{*}} R_{V} C_{2} & =L_{V^{*}} R_{V} P\left(L_{T_{2}}, R_{T_{2}^{*}}\right) C_{2} \\
& =L_{V^{*}} R_{V} Q\left(L_{T_{2}}, R_{T_{2}^{*}}\right) I \\
& =Q\left(L_{T_{1}}, R_{T_{1}^{*}}\right) L_{V^{*}} R_{V} I \\
& =Q\left(L_{T_{1}}, R_{T_{1}^{*}}\right) I,
\end{aligned}
$$

since $V^{*} V=I$. Thus $T_{1} \in \operatorname{dom} \Phi_{H_{1}}$ and $V^{*} C_{2} V \in \Phi_{H_{1}}\left(T_{1}\right)$, which is what we wanted to prove.

Corollary 4.4. Let $\Omega$ and $\mathcal{H}$ satisfy (4.1) and let $T$ be a commuting operator tuple on a separable Hilbert space $H$. Then the following are equivalent:

(a) $T^{*}$ is unitarily equivalent to the restriction to an invariant subspace of the direct sum of countably many copies of $Z^{*}$;

(b) there exists a nonnegative operator $C \in \mathcal{B}(\mathcal{H})$ such that

$$
P\left(L_{T}, R_{T^{*}}\right) C=Q\left(L_{T}, R_{T^{*}}\right) I
$$

and $f_{m, C}(T) \rightarrow 0$ strongly as $m \rightarrow \infty$. 


\section{REINHARDT DOMAINS AND THE UNITARY PART}

In this section we continue the study of the spaces from Section 4 under the additional hypothesis that both $\Omega$ and $\mathcal{H}$ are rotation invariant. In this case we are able to describe also the "unitary part", i.e., to obtain an analogue of the theorem (0.2).

Throughout this section, let thus $\Omega$ be a complete Reinhardt domain and assume that the space $\mathcal{H}$ is rotation invariant. This means that $z \in \Omega$ and $\left|x_{j}\right| \leq\left|z_{j}\right|$ $\forall j$ implies $x \in \Omega$ (in particular, $0 \in \Omega$ ) and that for any $\theta \in \mathbb{R}^{d}$, the function $U_{\theta} f(z):=f\left(e^{i \theta_{1}} z_{1}, \ldots, e^{i \theta_{d}} z_{d}\right)$ belongs to $\mathcal{H}$ if and only if $f$ does, and $\left\|U_{\theta} f\right\|_{\mathcal{H}}=$ $\|f\|_{\mathcal{H}}$. It follows easily that the monomials $z^{\alpha}, \alpha$ a multi-index, belonging to $\mathcal{H}$ form an orthogonal basis of $\mathcal{H}$, and for a function $f \in \mathcal{H}$ with Taylor expansion $f(z)=\sum_{\alpha} f_{\alpha} z^{\alpha}$,

$$
\|f\|_{\mathcal{H}}^{2}=\sum_{\alpha} \frac{\left|f_{\alpha}\right|^{2}}{\gamma_{\alpha}}
$$

where the sum extends over all multi-indices $\alpha=\left(\alpha_{1}, \ldots, \alpha_{d}\right), \alpha_{1}, \ldots, \alpha_{d} \geq 0$, and

$$
\gamma_{\alpha}:=\left\|z_{\alpha}\right\|^{-2}
$$

with the usual convention that $\gamma_{\alpha}=0$ if $z^{\alpha} \notin \mathcal{H}$. The reproducing kernel is given by

$$
K(x, \bar{y})=\sum_{\alpha} \gamma_{\alpha} x^{\alpha} \bar{y}^{\alpha}
$$

As in Section 4, we assume that $K$ is a rational function, i.e., is of the form (4.1). The rotational symmetry then implies that, in fact,

$$
K(x, \bar{y})=\frac{p(x \bar{y})}{q(x \bar{y})}, \quad x \bar{y}:=\left(x_{1} \bar{y}_{1}, \ldots, x_{d} \bar{y}_{d}\right) \in \mathbb{C}^{d},
$$

with holomorphic polynomials $p, q$ on $\mathbb{C}^{d}$ without a common factor, and such that $q(x \bar{x})>0 \forall x \in \Omega$. Denote by $p_{\alpha}, q_{\alpha}$ the Taylor coefficients of $p$ and $q$, respectively:

$$
p(z)=\sum_{\alpha} p_{\alpha} z^{\alpha}, \quad q(z)=\sum_{\alpha} q_{\alpha} z^{\alpha}
$$

(only finitely many of $p_{\alpha}, q_{\alpha}$ are nonzero). Observe that since $K(x, \bar{x}) \geq 0 \forall x \in \Omega$, all $p_{\alpha}$ and $q_{\alpha}$ are necessarily real. From (5.2), (5.3) and (5.4) it follows that for each $\alpha$,

$$
p_{\alpha}=\sum_{\beta+\delta=\alpha} \gamma_{\beta} q_{\delta}
$$

Recall finally that for a separable Hilbert space $H$ and $T \in \mathcal{B}(\mathcal{H}), C \in \mathcal{B}(\mathcal{H})^{+}$and $m \geq 0$, we have defined in (1.3) in Section 1 ,

$$
f_{m, C}(T)=I-\sum_{j=0}^{m-1} \psi_{j}(T) C \psi_{j}(T)^{*},
$$

where $\left\{\psi_{j}\right\}$ is an orthonormal basis of $\mathcal{H}$. Unless explicitly stated otherwise, from now on we will always take for $\left\{\psi_{j}\right\}$ the orthonormal basis $\left\{\gamma_{\alpha}^{1 / 2} z^{\alpha}\right\}$ of monomials lying in $\mathcal{H}$. Finally, for the sake of brevity, we will write $L_{T} R_{T^{*}}$ for the commuting $d$-tuple $\left(L_{T_{1}} R_{T_{1}^{*}}, \ldots, L_{T_{d}} R_{T_{d}^{*}}\right)$. Then we have the following assertion. 
Theorem 5.1. Let $\Omega, \mathcal{H}$ and $K$ be as above. Assume that $T \in \mathcal{B}(\mathcal{H})$ and $C \in$ $\mathcal{B}(\mathcal{H})^{+}$are such that

$$
p\left(L_{T} R_{T^{*}}\right) C=q\left(L_{T} R_{T^{*}}\right) I
$$

and

$$
f_{m, C}(T) \geq 0 \quad \text { for all } \quad m=0,1,2, \ldots .
$$

Assume further that

$$
q_{\alpha} \leq 0 \quad \text { if } \quad|\alpha|>0, \quad q_{\alpha}<0 \quad \text { if } \quad|\alpha|=1,
$$

where $|\alpha|=\alpha_{1}+\cdots+\alpha_{d}$; in other words, all coefficients of $q(0)-q$ are nonnegative, and the ones at the linear terms are even positive. Then there exists a Hilbert space $F$, an operator $W \in \mathcal{B}(F)$, and an isometry $U: H \rightarrow(\mathcal{H} \otimes H) \oplus F$ such that $W$ is a normal tuple,

$$
q\left(L_{W} R_{W^{*}}\right) I=0,
$$

and

$$
U T^{*}=[(Z \otimes I) \oplus W]^{*} U .
$$

By analogy with the $m$-contractions of Agler Ag2, we will call an operator $W$ satisfying (5.9) a $q$-isometry, and if in addition $W$ is a normal tuple (i.e., its elements are commuting normal operators) we will call it q-unitary.

Proof. Since

$$
f_{m, C}-f_{m+1, C}=\psi_{m}(T) C \psi_{m}(T)^{*} \geq 0
$$

owing to the positivity of $C$, it follows from (5.7) that there exists an operator $R \geq 0$ such that $\lim _{m \rightarrow \infty} f_{m, C}(T)=R$ in the strong operator topology; that is,

$$
R=I-\sum_{\alpha} \gamma_{\alpha} T^{\alpha} C T^{* \alpha}
$$

with the sum converging in SOT. For any multi-index $\beta$, we then have

$$
T^{\beta} R T^{* \beta}=T^{\beta} T^{* \beta}-\sum_{\alpha} \gamma_{\alpha} T^{\alpha+\beta} C T^{* \alpha+\beta} .
$$

Consequently,

$$
\begin{aligned}
q\left(L_{T} R_{T^{*}}\right) R & =\sum_{\beta} q_{\beta} T^{\beta} R T^{* \beta} \\
& =\sum_{\beta} q_{\beta} T^{\beta} T^{* \beta}-\sum_{\alpha, \beta} \gamma_{\alpha} q_{\beta} T^{\alpha+\beta} C T^{* \alpha+\beta} \\
& =\sum_{\beta} q_{\beta} T^{\beta} T^{* \beta}-\sum_{\delta} p_{\delta} T^{\delta} C T^{* \delta} \quad \text { by }(5.5) \\
& =q\left(L_{T} R_{T^{*}}\right) I-p\left(L_{T} R_{T^{*}}\right) C \\
& =0 \quad \text { by }(5.6) .
\end{aligned}
$$


By (5.8) this implies that

$$
\begin{aligned}
q(0) R & =-\sum_{|\alpha|>0} q_{\alpha} T^{\alpha} R T^{* \alpha} \geq-\sum_{|\alpha|=1} q_{\alpha} T^{\alpha} R T^{* \alpha} \\
& \geq c \sum_{j=1}^{d} T_{j} R T_{j}^{*}
\end{aligned}
$$

where $c=\min _{|\alpha|=1}\left(-q_{\alpha}\right)>0$. Thus there exists a commuting tuple $\widetilde{W}$ of bounded linear operators on $\widetilde{F}$, where $\widetilde{F}:=\overline{\operatorname{Ran} R^{1 / 2}}$, such that

$$
R^{1 / 2} T_{j}^{*}=\widetilde{W}_{j}^{*} R^{1 / 2}, \quad j=1,2, \ldots, d .
$$

From $q\left(L_{T} R_{T^{*}}\right) R=0$ we then get $q\left(L_{\widetilde{W}} R_{\widetilde{W}^{*}}\right) I=0$, i.e., $\widetilde{W}$ is a $q$-isometry on $\widetilde{F}$. Proceeding as in Pott [Po], Lemma 3.10 (using Athavale [At2], Proposition 2), we see that there exists a separable Hilbert space $F$ containing $\widetilde{F}$ and a $q$-unitary commuting tuple $W^{*}$ on $F$ which extends $\widetilde{W}^{*}$ (i.e., $\left.W^{*}\right|_{\widetilde{F}}=\widetilde{W}^{*}$ ). Let now $V$ : $H \rightarrow \mathcal{H} \otimes H$ be the operator (1.5) constructed in the proof of Theorem 1.3, viz.

$$
V h=\sum_{\alpha} \gamma_{\alpha} z^{\alpha} \otimes C^{1 / 2} T^{* \alpha} h,
$$

and set

$$
U=V \oplus R^{1 / 2} .
$$

As in the proof of Theorem 1.3, we have

$$
\|V h\|^{2}=\|h\|^{2}-\lim _{m \rightarrow \infty}\left\langle f_{m, C} h, h\right\rangle=\|h\|^{2}-\left\|R^{1 / 2} h\right\|^{2}
$$

and

thus,

$$
V T^{*}=(Z \otimes I)^{*} V
$$

$$
\|U h\|^{2}=\|V h\|^{2}+\left\|R^{1 / 2} h\right\|^{2}=\|h\|^{2},
$$

i.e., $U$ is an isometry, and, by (5.11),

$$
U T^{*}=V T^{*} \oplus R^{1 / 2} T^{*}=(Z \otimes I)^{*} V \oplus W^{*} R^{1 / 2}=[(Z \otimes I) \oplus W]^{*} U,
$$

which completes the proof.

Of course, the converse to the last theorem also holds, even without the hypothesis (5.8).

Theorem 5.2. Let $E, F, H$ be separable Hilbert spaces, $T$ a commuting operator tuple on $H, W$ a q-isometry on $F$, and $U: H \rightarrow(\mathcal{H} \otimes E) \oplus F$ an isometry such that $U T^{*}=[(Z \otimes I) \oplus W]^{*} U$. Then there exists an operator $C \in \mathcal{B}(\mathcal{H})^{+}$such that (5.6) and (5.7) are satisfied.

Proof. Denote, for brevity, $M=(Z \otimes I) \oplus W$ and set $C:=U^{*}\left[\left(P_{0} \otimes I\right) \oplus 0\right] U$.

Clearly $C$ is a nonnegative operator. As in the proof of Theorem 4.3, we then have

$$
\begin{aligned}
p\left(L_{T} R_{T^{*}}\right) C & =p\left(L_{T} R_{T^{*}}\right) L_{U^{*}} R_{U}\left[\left(P_{0} \otimes I\right) \oplus 0\right] \\
& =L_{U^{*}} R_{U} p\left(L_{M} R_{M^{*}}\right)\left[\left(P_{0} \otimes I\right) \oplus 0\right] \\
& =L_{U^{*}} R_{U}\left[\left(q\left(L_{Z} R_{Z^{*}}\right) I \otimes I\right) \oplus 0\right] \quad \text { by Propositon } 4.2 \\
& =L_{U^{*}} R_{U}\left[\left(q\left(L_{Z} R_{Z^{*}}\right) I \otimes I\right) \oplus q\left(L_{W} R_{W^{*}}\right) I\right] \\
& =L_{U^{*}} R_{U}\left[q\left(L_{M} R_{M^{*}}\right) I\right]=q\left(L_{T} R_{T^{*}}\right) I,
\end{aligned}
$$


where on the penultimate line we have used the fact that $W$ is a $q$-isometry. This proves (5.6). Recall that we have seen in the course of the proof of Theorem 1.3 that

$$
I_{\mathcal{H}}-\sum_{0 \leq j<m} \psi_{j}(Z) P_{0} \psi_{j}(Z)^{*}=: \pi_{m}, \quad \text { say, }
$$

is the orthogonal projection in $\mathcal{H}$ onto the span of $\left\{\psi_{j}\right\}_{j \geq m}$. By a similar argument as above, it therefore follows that, for our choice of $C$,

$$
\begin{aligned}
f_{m, C}(T) & =I-\sum_{0 \leq j<m} \psi_{j}(T) C \psi_{j}(T)^{*} \\
& =U^{*} U-\sum_{0 \leq j<m} U^{*} \psi_{j}(M)\left[\left(P_{0} \otimes I\right) \oplus 0\right] \psi_{j}(M)^{*} U \\
& =U^{*}\left[\left(\pi_{m} \otimes I\right) \oplus I\right] U \\
& \geq U^{*}[0 \oplus I] U \geq 0
\end{aligned}
$$

for any $m$; so (5.7) holds as well. This completes the proof.

In the case of $p \equiv 1$, the last two results have been obtained by a different method by Pott $\underline{\mathrm{Po}}$.

Remark. The hypothesis (5.8) is needed only to ensure that

$$
\exists c>0: \quad \sum_{j} T_{j} R T_{j}^{*} \leq c R
$$

(which in turn ensures the boundedness of the operator $\widetilde{W}$ ) and for extending the $q$-isometry $\widetilde{W}$ to a $q$-unitary $W$, but serves no other purpose. Hence if we are content with a $q$-isometry $W$, we can replace (5.8) by (5.12) in Theorem 5.1.

\section{The Unitary part on CARTAN Domains}

We conclude by establishing analogues of Theorems 5.1 and 5.2 for the standard "weighted Bergman" spaces $\mathcal{H}=\mathcal{H}_{\nu}$ on Cartan domains introduced in Section 2.

We will often use the following well-known fact without further mention:

Lemma 6.1. If $A_{1}, A_{2}, \ldots$ is a sequence of nonnegative operators, then $\sum A_{j}$ converges in the strong operator topology (SOT for short) if and only if

$$
\sup _{M \text { finite }}\left\|\sum_{j \in M} A_{j}\right\|<\infty
$$

if and only if some subsequence of its partial sums converges in SOT, if and only if some rearrangement of the series converges in SOT.

We will need the following technical result.

Proposition 6.2. Let $T$ be a commuting tuple of operators in $\mathcal{B}(\mathcal{H})$ and $c_{\mathbf{m}} \geq 0$ a sequence of nonnegative numbers, indexed by the partitions $\mathbf{m}$, such that

$$
\text { the sum } \sum_{\mathbf{m}} c_{\mathbf{m}} K_{\mathbf{m}}\left(T, T^{*}\right) \text { converges in SOT. }
$$

Then for each $X \in \mathcal{B}(\mathcal{H})$, the sum

$$
S(X):=\sum_{\mathbf{m}} c_{\mathbf{m}} K_{\mathbf{m}}\left(L_{T}, R_{T^{*}}\right) X
$$


converges in $S O T$ in $\mathcal{B}(\mathcal{H})$. Further, the resulting operator $S \in \mathcal{B}(\mathcal{B}(\mathcal{H})$ ) satisfies

$$
X_{m} \stackrel{\text { sot }}{\longrightarrow} X \Longrightarrow S X_{m} \stackrel{\text { sot }}{\rightarrow} S X
$$

Proof. Choose again, for each $\mathbf{m}$, an orthonormal (with respect to the Fock norm) basis $\left\{\psi_{\mathbf{m} j}\right\}_{j=1}^{d_{\mathbf{m}}}$ of $\mathcal{P}_{\mathbf{m}}$. Then

$$
K_{\mathbf{m}}\left(L_{T}, R_{T^{*}}\right) X=\sum_{j=1}^{d_{\mathbf{m}}} \psi_{\mathbf{m} j}(T) X \psi_{\mathbf{m} j}(T)^{*}
$$

Hence for any $X \in \mathcal{B}(\mathcal{H})^{+}$and any finite set $M$ of signatures,

$$
\sum_{\mathbf{m} \in M} \sum_{j=1}^{d_{\mathbf{m}}} c_{\mathbf{m}} \psi_{\mathbf{m} j}(T) X \psi_{\mathbf{m} j}(T)^{*}=\mathbf{B}_{M} \mathbf{X B}_{M}^{*}
$$

where $\mathbf{B}_{M}$ is the row block operator $\mathbf{B}_{M}=\left[\sqrt{c_{\mathbf{m}}} \chi_{M}(\mathbf{m}) \psi_{\mathbf{m} j}(T)\right]_{\mathbf{m}, j}$, with $\chi_{M}$ the characteristic function of the set $M$, and $\mathbf{X}=\operatorname{diag}(X)$ is the block matrix operator with $X$ 's on the main diagonal and zeroes elsewhere. Since both $\psi_{\mathbf{m} j}(T) X \psi_{\mathbf{m} j}(T)^{*}$ and $\psi_{\mathbf{m} j}(T) \psi_{\mathbf{m} j}(T)^{*}$ are nonnegative operators, and

$$
\left\|\mathbf{B}_{M} \mathbf{X B}_{M}^{*}\right\| \leq\left\|\mathbf{B}_{M}\right\|^{2}\|\mathbf{X}\|=\left\|\mathbf{B}_{M} \mathbf{B}_{M}^{*}\right\|\|X\|=\left\|\sum_{\mathbf{m} \in M} c_{\mathbf{m}} K_{\mathbf{m}}\left(T, T^{*}\right)\right\| \cdot\|X\|,
$$

it transpires from Lemma 6.1 that for the sum (6.2) to converge in SOT it is sufficient that (6.1) hold. (Incidentally, since the sum in (6.1) is just $S(I)$, this condition is also necessary.) Since any $X \in \mathcal{B}(\mathcal{H})$ can be expressed as $X=X_{1}-$ $X_{2}+i\left(X_{3}-X_{4}\right)$ with $X_{1}, X_{2}, X_{3}, X_{4} \in \mathcal{B}(\mathcal{H})^{+}$, the first assertion of the proposition follows, and we also see that $S X$ can be expressed as

$$
S X=\mathbf{B X B}^{*},
$$

with the row block operator $\mathbf{B}=\left[\sqrt{c_{\mathbf{m}}} \psi_{\mathbf{m} j}(T)\right]_{\mathbf{m}, j}$ bounded from $\mathbb{H}:=\bigoplus_{\mathbf{m}} \bigoplus_{j=1}^{d_{\mathbf{m}}} H$ into $H\left(\|\mathbf{B}\|^{2}=\left\|\mathbf{B B}^{*}\right\|=\left\|\sum_{\mathbf{m}} c_{\mathbf{m}} K_{\mathbf{m}}\left(T, T^{*}\right)\right\|\right)$. Thus if $X_{m} \stackrel{\text { sot }}{\rightarrow} X$ in $\mathcal{B}(\mathcal{H})$, whence also $\mathbf{X}_{m} \stackrel{\text { sot }}{\rightarrow} \mathbf{X}$ in $\mathcal{B}(\mathbb{H})$, we have $\mathbf{B X}_{m} \mathbf{B}^{*} \stackrel{\text { sot }}{\rightarrow} \mathbf{B X B}^{*}$ in $\mathcal{B}(\mathcal{H})$, i.e., $S X_{m} \stackrel{\text { sot }}{\rightarrow}$ $S X$ in $\mathcal{B}(\mathcal{H})$.

Remark. The proof above shows, in particular, that

$$
\left\|K_{\mathbf{m}}\left(L_{T}, R_{T^{*}}\right)\right\|_{\mathcal{B}(\mathcal{B}(\mathcal{H}))}=\left\|K_{\mathbf{m}}\left(T, T^{*}\right)\right\|_{\mathcal{B}(\mathcal{H})}
$$

an equality which is interesting in its own right.

Modifying the definition from Section 1 in a trivial manner, let us define, for $T$ a commuting operator tuple on a separable Hilbert space $H$ and $C \in \mathcal{B}(\mathcal{H})^{+}$,

$$
f_{m, C}(T):=I-\sum_{|\mathbf{m}|<m}(\nu)_{\mathbf{m}} K_{\mathbf{m}}\left(L_{T}, R_{T^{*}}\right) C .
$$

Observe that, for any $\nu>0$,

$$
f_{m, C}-f_{m+1, C}=\sum_{|\mathbf{m}|=m}(\nu)_{\mathbf{m}} K_{\mathbf{m}}\left(L_{T}, R_{T^{*}}\right) C \geq 0 .
$$

So if $f_{m, C} \geq 0 \forall m$, then $f_{m, C} \searrow R$ for some $R \geq 0$, in the strong operator topology.

We can now state our two main results of this section. 
Theorem 6.3. Let $H$ be a separable Hilbert space, $\nu>\frac{r-1}{2} a$, and $T$ a commuting operator tuple on $H$ such that

$$
\sum_{\mathbf{k}}\left|(-\nu)_{\mathbf{k}}\right| K_{\mathbf{k}}\left(T, T^{*}\right) \quad \text { converges strongly. }
$$

Set $C:=\operatorname{sot}-\sum_{\mathbf{k}}(-\nu)_{\mathbf{k}} K_{\mathbf{k}}\left(T, T^{*}\right)$. Assume further that $f_{m, C}(T) \geq 0 \forall m$, so that $R:=$ sot- $\lim _{m \rightarrow \infty} f_{m, C}$ exists and $R \geq 0$. Assume finally that there exists a $c>$ 0 such that $\sum_{j} T_{j} R T_{j}^{*} \leq c R$. Then there exists a separable Hilbert space $F$, a commuting operator tuple $W$ on $F$ and an isometry $U: H \rightarrow(\mathcal{H} \otimes H) \oplus F$ such that

(i) $\sum_{\mathbf{k}}\left|(-\nu)_{\mathbf{k}}\right| K_{\mathbf{k}}\left(W, W^{*}\right)$ converges strongly, and $\sum_{\mathbf{k}}(-\nu)_{\mathbf{k}} K_{\mathbf{k}}\left(W, W^{*}\right)=0$;

(ii) $U T^{*}=[(Z \otimes I) \oplus W]^{*} U$.

Proof. From Proposition 6.2 we see that the sums

$$
S_{ \pm} X:=\sum_{\left\{\mathbf{m}: \pm(-\nu)_{\mathbf{m}}>0\right\}}(-\nu)_{\mathbf{m}} K_{\mathbf{m}}\left(L_{T}, R_{T^{*}}\right) X \quad \text { and } \quad S:=S_{+}-S_{-}
$$

converge strongly for each $X \in \mathcal{B}(\mathcal{H})$ (in particular, $C=S I$ ), and that $X_{m} \stackrel{\text { sot }}{\rightarrow} X$ implies $S_{+} X_{m} \stackrel{\text { sot }}{\rightarrow} S_{+} X$ and similarly for $S_{-}$and $S$. Applying this to $X_{m}=$ $\sum_{|\mathbf{m}|<m}(\nu)_{\mathbf{m}} K_{\mathbf{m}}\left(L_{T}, R_{T^{*}}\right) C$, so that $X=I-R$, we thus obtain

$$
\begin{aligned}
S_{+}(R) & =S_{+}(I)-S_{+}(X) \\
& =S_{+}(I)-\lim _{m \rightarrow \infty} S_{+}\left(X_{m}\right) \\
& =S_{+}(I)-\lim _{m \rightarrow \infty} \sum_{\left\{\mathbf{j}:(-\nu)_{\mathbf{j}}>0\right\}}\left|(-\nu)_{\mathbf{j}}\right| K_{\mathbf{j}}\left(L_{T}, R_{T^{*}}\right) \sum_{|\mathbf{m}|<m}(\nu)_{\mathbf{m}} K_{\mathbf{m}}\left(L_{T}, R_{T^{*}}\right) C \\
& =S_{+}(I)-\sum_{\mathbf{m}} \sum_{\left\{\mathbf{j}:(-\nu)_{\mathbf{j}}>0\right\}}(\nu)_{\mathbf{m}} K_{\mathbf{m}}\left(L_{T}, R_{T^{*}}\right)\left|(-\nu)_{\mathbf{j}}\right| K_{\mathbf{j}}\left(L_{T}, R_{T^{*}}\right) C
\end{aligned}
$$

(all the sums and limits are in SOT). By Lemma 6.1, we can rearrange the last double sum into $\sum_{l \geq 0} \sum_{|\mathbf{m}|+|\mathbf{j}|=l,(-\nu)_{\mathbf{j}}>0}$, and similarly for $S_{-}$. Upon subtracting, we thus obtain

$$
\begin{aligned}
S(R) & =S(I)-\sum_{l \geq 0} \sum_{|\mathbf{m}|+|\mathbf{j}|=l}(\nu)_{\mathbf{m}}(-\nu)_{\mathbf{j}} K_{\mathbf{m}}\left(L_{T}, R_{T^{*}}\right) K_{\mathbf{j}}\left(L_{T}, R_{T^{*}}\right) C \\
& =C-\sum_{l \geq 0} \sum_{|\mathbf{k}|=l}(0)_{\mathbf{k}} K_{\mathbf{k}}\left(L_{T}, R_{T^{*}}\right) C \quad \text { by }(2.9) \text { and Proposition } 3.7 \\
& =C-C=0 .
\end{aligned}
$$

Owing to the hypothesis $\sum_{j} T_{j} R T_{j}^{*} \leq c R$, or

$$
\left[T_{1}, \ldots, T_{d}\right] R\left[T_{1}, \ldots, T_{d}\right]^{*} \leq c R,
$$

there exists a commuting tuple $W$ of bounded operators on $F:=\overline{\operatorname{Ran} R^{1 / 2}}$ such that $W^{*} R^{1 / 2}=R^{1 / 2} T^{*}$. Consequently, $K_{\mathbf{m}}\left(L_{T}, R_{T^{*}}\right) R=R^{1 / 2} K_{\mathbf{m}}\left(L_{W}, R_{W^{*}}\right) R^{1 / 2}$; thus (6.4) implies that

$$
\sum_{\mathbf{m}}\left|(-\nu)_{\mathbf{m}}\right| K_{\mathbf{m}}\left(L_{W}, R_{W^{*}}\right) I
$$

converges strongly in $\mathcal{B}(F)$, and $S(R)=0$ implies that

$$
\sum_{\mathbf{m}}(-\nu)_{\mathbf{m}} K_{\mathbf{m}}\left(W, W^{*}\right)=0
$$


so we have proved (i). Finally, let $V: H \rightarrow \mathcal{H} \otimes H$ be the operator (1.5) constructed in the proof of Theorem 1.3, viz.

$$
V=\sum_{\mathbf{m}}(\nu)_{\mathbf{m}} K_{\mathbf{m}}\left(z, R_{T^{*}}\right) C^{1 / 2} .
$$

As in the proof of Theorem 1.3, one shows that $V T^{*}=(Z \otimes I)^{*} V$ and

$$
\|V h\|^{2}=\|h\|^{2}-\left\|R^{1 / 2} h\right\|^{2} .
$$

Hence

$$
U:=V \oplus R^{1 / 2}
$$

is an isometry with the required property (ii). The proof is complete.

Of course, the converse to the last theorem is also true. Recall from Section 3 that the condition (6.5) below is always satisfied for $\nu \geq d / r$, and for the ball even for all $\nu>0$ (cf. Propositions 3.4, 3.5 and 3.9).

Theorem 6.4. If $\nu$ is such that

$$
\sup _{\mathbf{m}}\left[\sum_{\mathbf{k}}\left|(-\nu)_{\mathbf{k}}\right| \tau_{\mathbf{k}}(\mathbf{m})\right]<\infty,
$$

$E, F, H$ are separable Hilbert spaces, $W$ a commuting operator tuple on $F$ such that

$$
\sum_{\mathbf{k}}\left|(-\nu)_{\mathbf{k}}\right| K_{\mathbf{k}}\left(W, W^{*}\right)
$$

converges strongly and

$$
\sum_{\mathbf{k}}(-\nu)_{\mathbf{k}} K_{\mathbf{k}}\left(W, W^{*}\right)=0,
$$

and $U: H \rightarrow(\mathcal{H} \otimes E) \oplus F$ an isometry such that

$$
U T^{*}=[(Z \otimes I) \oplus W]^{*} U,
$$

then $\sum_{\mathbf{k}}\left|(-\nu)_{\mathbf{k}}\right| K_{\mathbf{k}}\left(T, T^{*}\right)$ converges strongly, and setting

$$
C:=\operatorname{sot}-\sum_{\mathbf{k}}(-\nu)_{\mathbf{k}} K_{\mathbf{k}}\left(T, T^{*}\right),
$$

we have $C \in \mathcal{B}(\mathcal{H})^{+}, f_{m, C}(T) \searrow R \geq 0$ as $m \rightarrow \infty$, and the limit $R$ satisfies $T R T^{*} \leq\|W\|^{2} R$.

Proof. Since $K_{\mathbf{k}}\left(Z, Z^{*}\right)$ is a diagonal operator with respect to the Peter-Weyl decomposition (2.1) with weights $\tau_{\mathbf{k}}(\mathbf{m})$, the condition (6.5) is equivalent to the strong convergence of the series (6.4) for $T=Z$. Using (6.8), it therefore follows from (6.6) that $\sum_{\mathbf{m}}\left|(-\nu)_{\mathbf{m}}\right| K_{\mathbf{m}}\left(T, T^{*}\right)$ converges strongly, and from (6.7) and Corollary 3.8 that

$$
C=\sum_{\mathbf{m}}(-\nu)_{\mathbf{m}} K_{\mathbf{m}}\left(T, T^{*}\right)=U^{*}\left[\left(P_{0} \otimes I\right) \oplus 0\right] U .
$$

As in the proof of Theorem 5.2, we therefore obtain

$$
f_{m, C}(T)=U^{*}\left[\left(\pi_{m} \otimes I\right) \oplus I\right] U
$$

with $\pi_{m}$ the projection in $\mathcal{H}$ onto $\bigoplus_{|\mathbf{m}| \geq m} \mathcal{P}_{\mathbf{m}}$. Hence $f_{m, C} \geq 0 \forall m$ and

$$
\lim _{m \rightarrow \infty} f_{m, C}=R=U^{*}[0 \oplus I] U .
$$


It follows that

$$
\begin{aligned}
T R T^{*} & =T U^{*}[0 \oplus I] U T^{*} \\
& =U^{*}[(Z \otimes I) \oplus W][0 \oplus I][(Z \otimes I) \oplus W]^{*} U \\
& =U^{*}\left[0 \oplus W W^{*}\right] U \\
& \leq U^{*}\left[0 \oplus\|W\|^{2} I\right] U=\|W\|^{2} R,
\end{aligned}
$$

and the proof is complete.

\section{REFERENCES}

[Ag1] J. Agler, The Arveson extension theorem and coanalytic models, Integ. Equations Oper. Theory 5 (1982), 608-631. MR 84g:47011

[Ag2] J. Agler, Hypercontractions and subnormality, J. Oper. Theory 13 (1985), 203-217. MR 86i: 47028

[AEM] C. Ambrozie, M. Engliš, V. Müller, Operator tuples and analytic models over general domains in $\mathbb{C}^{n}$, preprint (1999), J. Oper. Theory 47 (2002), 287-302.

[Ara] J. Arazy, A survey of invariant Hilbert spaces of analytic functions on bounded symmetric domains, Multivariable operator theory (R.E. Curto, R.G. Douglas, J.D. Pincus, N. Salinas, eds.), Contemporary Mathematics, vol. 185, Amer. Math. Soc., Providence, 1995, pp. 7-65. MR 96e:46034

[ArU] J. Arazy, H. Upmeier, Boundary integration and the discrete Wallach points, ESI preprint No. 762 (1999), http://www.esi.ac.at/preprints/ESI-Preprints.html, to be submitted to AMS Memoirs.

[ArZ] J. Arazy, G. Zhang, Homogeneous multiplication operators on bounded symmetric domains, preprint, 2001, submitted to J. Funct. Anal.

[Aro] N. Aronszajn, Theory of reproducing kernels, Trans. Amer. Math. Soc. 68 (1950), 337-404. MR 14:479c

[Arv] W. Arveson, Subalgebras of $C^{*}$-algebras III: Multivariable operator theory, Acta Math. 181 (1998), 159-228. MR 2000e:47013

[At1] A. Athavale, Holomorphic kernels and commuting operators, Trans. Amer. Math. Soc. 304 (1987), 101-110. MR 88m:47039

[At2] A. Athavale, On the intertwining of joint isometries, J. Oper. Theory 23 (1990), 339-350. MR 91i: 47029

[At3] A. Athavale, Model theory on the unit ball in $\mathbb{C}^{m}$, J. Oper. Theory 27 (1992), 347-358. MR 94i:47011

[Be] S. Bergman, The kernel function and conformal mapping, 2nd edition, AMS, Providence, 1970. MR 58:22502

[Cu] R. E. Curto, The spectra of elementary operators, Indiana Univ. Math. J. 32 (1983), 193-197. MR 84e:47005

[Dr] S. W. Drury, A generalization of von Neumann's inequality to the complex ball, Proc. Amer. Math. Soc. 68 (1978), 300-304. MR 80c:47010

[FK] J. Faraut, A. Koranyi, Function spaces and reproducing kernels on bounded symmetric domains, J. Funct. Anal. 88 (1990), 64-89. MR 90m:32049

[Fe] C. Fefferman, The Bergman kernel and biholomorphic mappings of pseudoconvex domains, Invent. Math. 26 (1974), 1-65. MR 50:2562

[Gh] C. R. Graham, Scalar boundary invariants and the Bergman kernel, Complex Analysis, II (College Park, MD, 1985-86), Lecture Notes in Math., vol. 1276, Springer, Berlin, 1987, pp. 108-135. MR 89d:32045

[KS] F. Knop, S. Sahi, A recursion and a combinatorial formula for Jack polynomials, Invent. Math. 128 (1997), 9-22. MR 98k:33040

[La] M. Lassalle, Une formule de Pieri pour les polynômes de Jack, C. R. Acad. Sci. Paris Sér. I Math. 309 (1989), 941-944 (French). MR 91c:05192

[Lo] O. Loos, Bounded symmetric domains and Jordan pairs, University of California, Irvine, 1977.

[MD] I. G. MacDonald, Symmetric functions and Hall polynomials, 2nd edition, Clarendon Press, Oxford, 1995. MR 96h:05207 
[M] V. P. Maslov, Operator Methods, Nauka, Moscow, 1973 (in Russian); English translation: Operational Methods, Mir, Moscow, 1976. MR 56:3647

[MN] V. P. Maslov, V. E. Nazaikinskii, Asymptotics of Operators and Pseudo-Differential Equations, Consultants Bureau, New York-London, 1988. MR 89i:58141

[MV] V. Müller, F.-H. Vasilescu, Standard models for some commuting multioperators, Proc. Amer. Math. Soc. 117 (1993), 979-989. MR 93e:47016

[Po] S. Pott, Standard models under polynomial positivity conditions, J. Oper. Theory 41 (1999), 365-389. MR 2000j:47019

[RV] H. Rossi, M. Vergne, Analytic continuation of the holomorphic discrete series of a semisimple Lie group, Acta Math. 136 (1976), 1-59. MR 58:1032

[St] R. Stanley, Some combinatorial properties of Jack symmetric functions, Adv. Math. $\mathbf{7 7}$ (1989), 76-115. MR 90g:05020

[Up] H. Upmeier, Toeplitz operators on bounded symmetric domains, Trans. Amer. Math. Soc. 280 (1983), 221-237. MR 85g:47042

[Vr] L. Vretare, Recurrence formulas for zonal polynomials, Math. Z. 188 (1985), 419-425. MR 86j:22012

[Zh] G. Zhang, Some recurrence formulas for spherical polynomials on tube domains, Trans. Amer. Math. Soc. 347 (1995), 1725-1734. MR 95h:22018

Department of Mathematics, University of Haifa, Haifa 31905, Israel

E-mail address: jarazy@math.haifa.ac.il

MÚ AV ČR, Žitná 25, 11567 Prague 1, Czech Republic

E-mail address: englis@math.cas.cz

URL: http://www.math.cas.cz/〜englis/ 